\title{
The Fundamental Role of General Orthonormal Bases in System Identification
}

\author{
Brett Ninness, Member, IEEE, Håkan Hjalmarsson, Member, IEEE, and Fredrik Gustafsson
}

\begin{abstract}
The purpose of this paper is threefold. Firstly, it is to establish that contrary to what might be expected, the accuracy of well-known and frequently used asymptotic variance results can depend on choices of fixed poles or zeros in the model structure. Secondly, it is to derive new variance expressions that can provide greatly improved accuracy while also making explicit the influence of any fixed poles or zeros. This is achieved by employing certain new results on generalized Fourier series and the asymptotic properties of Toeplitz-like matrices in such a way that the new variance expressions presented here encompass pre-existing ones as special cases. Via this latter analysis a new perspective emerges on recent work pertaining to the use of orthonormal basis structures in system identification. Namely, that orthonormal bases are much more than an implementational option offering improved numerical properties. In fact, they are an intrinsic part of estimation since, as shown here, orthonormal bases quantify the asymptotic variability of the estimates whether or not they are actually employed in calculating them.
\end{abstract}

Index Terms-Parameter estimation, system identification.

\section{INTRODUCTION}

A RESULT that has found great utility in practical applications of least squares system identification methods [8], [21], [2] is that the sensitivity to measurement noise of the ensuing frequency response estimate $\hat{G}\left(e^{j \omega}\right)$ may be quantified as [25], [22], [24], [50]

$$
\operatorname{Var}\left\{\hat{G}\left(e^{j \omega}\right)\right\} \approx \frac{n}{N} \frac{\Phi_{\nu}(\omega)}{\Phi_{u}(\omega)}
$$

where $\Phi_{\nu}(\omega)$ and $\Phi_{u}(\omega)$ are the measurement noise and input excitation spectral densities (respectively), $N$ is the length of the available data record, and $n$ is the order of the model $\hat{G}\left(e^{j \omega}\right)$.

In [25], [22], and [24] an essential principle in deriving (1) is to establish that

$$
\lim _{N, n \rightarrow \infty} \frac{N}{n} \operatorname{Var}\left\{\hat{G}\left(e^{j \omega}\right)\right\}=\frac{\Phi_{\nu}(\omega)}{\Phi_{u}(\omega)}
$$

Manuscript received June 21, 1996; revised November 28, 1997 and August 12, 1998. Recommended by Associate Editor, T. Katayama. This work was supported by the Australian Research Council and the Centre for Integrated Dynamics and Control (CIDAC), University of Newcastle, Callaghan 2308, Australia. Part of this work was completed while B. Ninness was visiting S3Automatic Control, The Royal Institute of Technology, Stockholm, Sweden. The work of H. Hjalmarsson and F. Gustafsson was partly completed while on leave at CIDAC.

B. Ninness is with CIDAC and the Department of Electrical \& Computer Engineering, University of Newcastle, Australia.

H. Hjalmarsson is with the Department of Sensors, Signals and Systems, The Royal Institute of Technology, S-100 44 Stockholm, Sweden.

F. Gustafsson is with the Department of Electrical Engineering, Linköping University, S-581 83, Linköping, Sweden.

Publisher Item Identifier S 0018-9286(99)05463-X. and then assume that in fact (2) holds approximately for finite $N$ and $n$, hence the result (1).

A main contribution of this paper is to show that in fact, for several commonly occurring situations, the approximation (1) can be quite misleading, even for large $N$ and $n$. For example, problems are shown to occur when employing model structures with fixed denominator terms or when utilizing extensions of ARX structures with fixed noise model zeros.

Given these new observations, a more important purpose of this work is to establish how (1) should be modified so as to provide improved accuracy. In deriving these enhanced accuracy expressions, the third and final main theme of this paper emerges, namely, to provide a new perspective on the role of orthonormal model structures in system identification.

To elaborate more on this final point, there has recently been significant activity on the system identification application of special classes of fixed denominator model structures that are constructed to be orthonormal; see for example [44], [45], [15], [37], [34], and the references therein. This latter work has been motivated by two main factors: firstly, the achievement of improved numerical conditioning and secondly, the provision of efficient parameterizations that allow decreased variance error while still minimizing bias error.

However, this second feature is not dependent on the orthonormal property of the model structure. This is so since the orthonormal structure is, under a linear parameter space transform, equivalent to any other equivalently flexible nonorthonormal structure with the same fixed poles. As well, since least squares methods are employed, the estimates depend linearly on the output measurements. Therefore, modulo numerical issues, precisely the same estimates of the system frequency response, are obtained whether or not one goes to the effort of implementing an orthonormal structure as opposed to a simpler structure wherein (for example) the denominator is fixed, and only the numerator coefficients are estimated.

Given this observation, a key contribution of this paper is to discover a new role for orthonormal bases in a system identification context that is beyond their implementational advantage of improved numerical conditioning. This insight, which involves exposing that orthonormal bases play a far more fundamental role in system identification theory than has been previously suggested, is achieved by taking a different approach to that of previous work [44], [45], [15], [37], [34] where only model structures parameterized in terms of orthonormal bases have been considered. In contrast, it is shown here how orthonormal bases may also be used as an 
analysis tool to determine the properties of estimates derived with respect to any model structure.

In particular, this strategy achieves the aforementioned goal of improving the accuracy of (1) by replacing $n$ in (1) with a function $K_{n}(\omega, \omega)$ which is the so-called "reproducing kernel" associated with a particular $n$-dimensional subspace and which depends on fixed poles or zeros. Note that even if such fixed components are not explicitly present in the model structure, they are implicitly there whenever the common practice of data prefiltering is employed; see Section VII.

As well, by utilizing the orthonormal basis formulation of [34], an explicit formula for $K_{n}(\omega, \omega)$ can be generated which clearly shows how $K_{n}(\omega, \omega)$, and hence $\operatorname{Var}\left\{\hat{G}\left(e^{j \omega}\right)\right\}$, is affected by fixed model structure poles or zeros. For example, it will be seen that in the special case of all the poles/zeros fixed at the origin (FIR/ARX model structures) $K_{n}(\omega, \omega)=n$, and hence (1) arises as a special case. However, if all the poles are not chosen at the origin, then inclusion of the $K_{n}(\omega, \omega)$ term in place of $n$ in (1) is shown here to smoothly extend the original analysis of [22], [25] and in so doing provide more accurate approximation of $\operatorname{Var}\left\{\hat{G}\left(e^{j \omega}\right)\right\}$ over a wider range of circumstances.

The paper is structured as follows. In Section II a short simulation example demonstrating the possible inaccuracy of the variance error approximation (1) is presented to motivate the ensuing analysis. The paper then provides a formal problem definition in Section III and in so doing allows Section IV to theoretically examine and hence isolate the cause of the inaccuracy demonstrated numerically in Section II. This leads to the work of Section V where it is shown how new techniques, based on new system theoretic results pertaining to generalized orthonormal bases, generalized Fourier convergence, and generalized Toeplitz-like matrices may be employed to provide a variance error approximation which, in some circumstances (such as those illustrated in Section II), is a great improvement over the pre-existing one (1). In Section VI it is shown how the same new tools may be used to also quantify the bias error for fixed denominator model structures. By the end of Section VI the paper has exclusively focused on fixed denominator generalizations of the FIR structure, but in Section VII the scope is broadened to also consider ARX-like structures with fixed zeros in the noise model. In analogy with the preceding analysis, it is demonstrated that if all the noise model zeros are not fixed at the origin, then the asymptotic variance expressions derived in [22] and [24], although prima facie applicable, are also liable to suffer from inaccuracy, the severity of which depends on the proportion of nonorigin placed zeros. Motivated by this, it is demonstrated in Section VII how the same new techniques and results of previous sections can be brought to bear in the ARX modeling context so as to provide new approximations whose validity is not degraded by choosing noise model zeros away from the origin. Finally, Section VIII provides some concluding perspectives on the results and techniques of the paper.

There have been a number of contributions dealing with certain aspects of the problems considered in this paper, the foremost of which are [25], [22], [44], [45], [15], [37], and
[10]. The sequel will comment on how these foundations relate to the paper at hand.

\section{Motivation}

In the interests of clearly motivating the analysis of this paper, consider the simple case of estimating, via least-squares, an $n=12$ th-order FIR model of the true system (the zeroorder hold equivalence is calculated using a sampling period of $1 \mathrm{~s}$ )

$$
\begin{aligned}
G(q) & =\mathcal{Z} \mathcal{O H}\left\{\frac{1}{(s+1)(2 s+1)}\right\} \\
& =\frac{0.1548 q+0.0939}{(q-0.6065)(q-0.3679)}
\end{aligned}
$$

by observing 10000 samples of its input-output response when the former is a stationary Gaussian process with spectral density $\Phi_{u}(\omega)=0.25 /(1.25-\cos \omega)$ and the latter is corrupted by zero mean Gaussian white noise of variance $\sigma^{2}=0.001$. In this case, since both $n$ and $N$ can reasonably be considered "large," then the approximation (1) could be expected to hold. This can be checked by Monte Carlo simulation over, say, 500 input and noise realizations so as to estimate the variance $\operatorname{Var}\left\{\hat{G}\left(e^{j \omega}\right)\right\}$ by its sample average, which can then be compared to the approximation (1). The results for just such an experiment are shown in Fig. 1 with the agreement between (dashed-dot line) the expression (1) and the sample average (solid line) being excellent. Note that in this simulation (and in all the rest to follow in this section), the bias error is negligible, and hence the variance error represents the total error.

Now suppose, as may commonly occur in practice, that prior knowledge of the poles of $G(q)$ exists. Then in the interests of decreasing the bias error it makes sense to try to incorporate this prior knowledge in the estimation process by fixing some poles in the model near where it is believed the true poles lie [44], [15]. For example, suppose in the previous simulation it is believed that a dominant pole is near $z=0.75$, so that guesses of, say, $z=\{0.7,0.72,0.78,0.8\}$ are to be incorporated into the model structure. This can be implemented by simply prefiltering the input by $F(q)=$ $q^{4} /(q-0.7)(q-0.72)(q-0.78)(q-0.8)$ before an FIR "numerator" model $\hat{G}(q)$ is estimated, and then the complete system $\hat{G}^{\prime}(q)=\hat{G}(q) F(q)$ may be taken as the fixed-pole estimate of the underlying dynamics.

Since the model order is still large $(n=12)$, then (1) should still provide a quantification of the variability of this new estimate by labeling the FIR estimate as $\hat{G}$ and reasoning

$$
\begin{aligned}
\operatorname{Var}\left\{\hat{G}^{\prime}\left(e^{j \omega}\right)\right\} & =\left|F\left(e^{j \omega}\right)\right|^{2} \operatorname{Var}\left\{\hat{G}\left(e^{j \omega}\right)\right\} \\
& \approx \frac{n}{N}\left|F\left(e^{j \omega}\right)\right|^{2} \frac{\Phi_{\nu}(\omega)}{\left|F\left(e^{j \omega}\right)\right|^{2} \Phi_{u}(\omega)} \\
& =\frac{n}{N} \frac{\Phi_{\nu}(\omega)}{\Phi_{u}(\omega)}
\end{aligned}
$$

which is unchanged from the normal FIR case. This unchanging nature is also reasonable, since the FIR case can be considered as already incorporating prior knowledge of system poles; namely poles near the origin. 


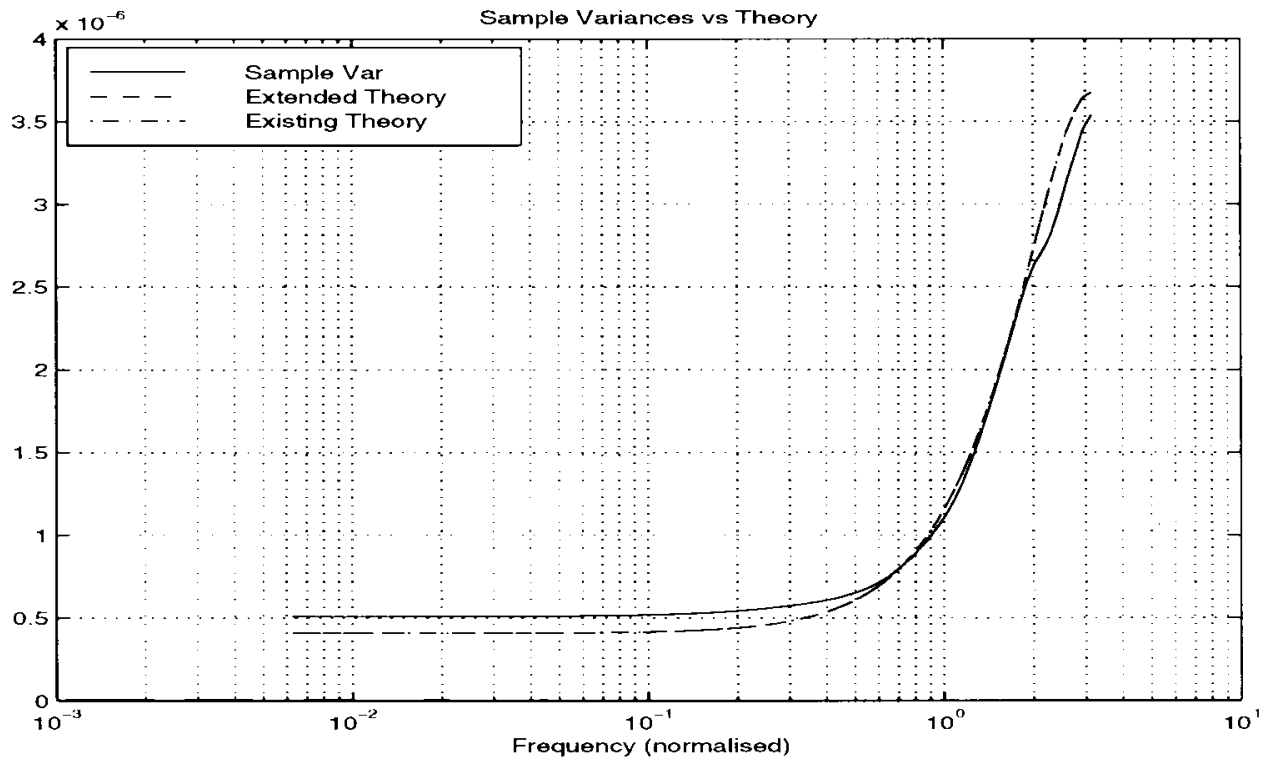

Fig. 1. FIR with all poles at origin. This is a comparison of a Monte Carlo estimate of sample variability (solid line) with (dashed-dot line) the approximate expression (1). Note that this last line obscures a dashed line which is the new approximation (53). The obfuscation occurs because for the case of all poles at the origin, the pre-existing approximation (1) and the new one (53) are identical.

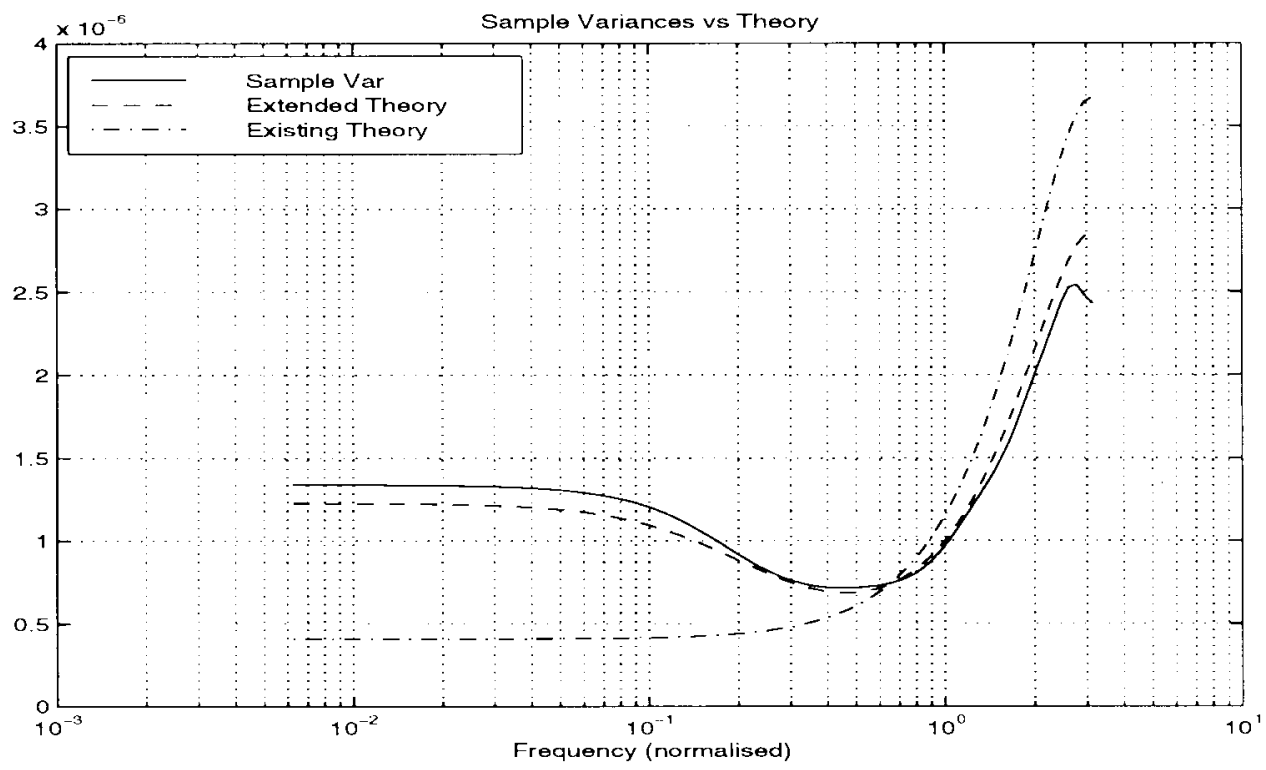

Fig. 2. FIR with four poles away from origin. This is a comparison of the Monte Carlo estimate of sample variability (solid line) with (dash-dot line) the approximate expression (1) and (dashed line) the new improved approximation (53) derived in this paper.

Interestingly, when the expression (3) is compared to Monte Carlo calculated sample variability as it is in Fig. 2, then the agreement between the true variability (solid line) and approximation (3) (dash-dot line) is seen to be not nearly as good as is Fig. 1. Nevertheless, the expression (3) still provides useful information on the qualitative "high-pass" nature of how the true variability changes with frequency. The dashed line near the solid one in Fig. 2 will be commented on in a moment.

Now suppose even more guesses of system poles are made, say, at the locations $z=$ $\{0.7,0.72,0.78,0.8,0.75,0.85,0.82,0.79\} \quad$ with the sample variability again being compared to (3) in Fig. 3. In this case there is virtually no agreement (even qualitative) between true and predicted variability.

Clearly then, the well-known approximation (1) can be quite misleading in situations where it would be expected to be reliable, and this apparently puzzling and practically important phenomenon is the motivation for this paper. In the work to follow, the conundrum just illustrated will be resolved by exposing certain hitherto unappreciated principles pertaining to when it is advisable to conclude (1) on the basis of (2). Furthermore, a new expression to extend (1) will 


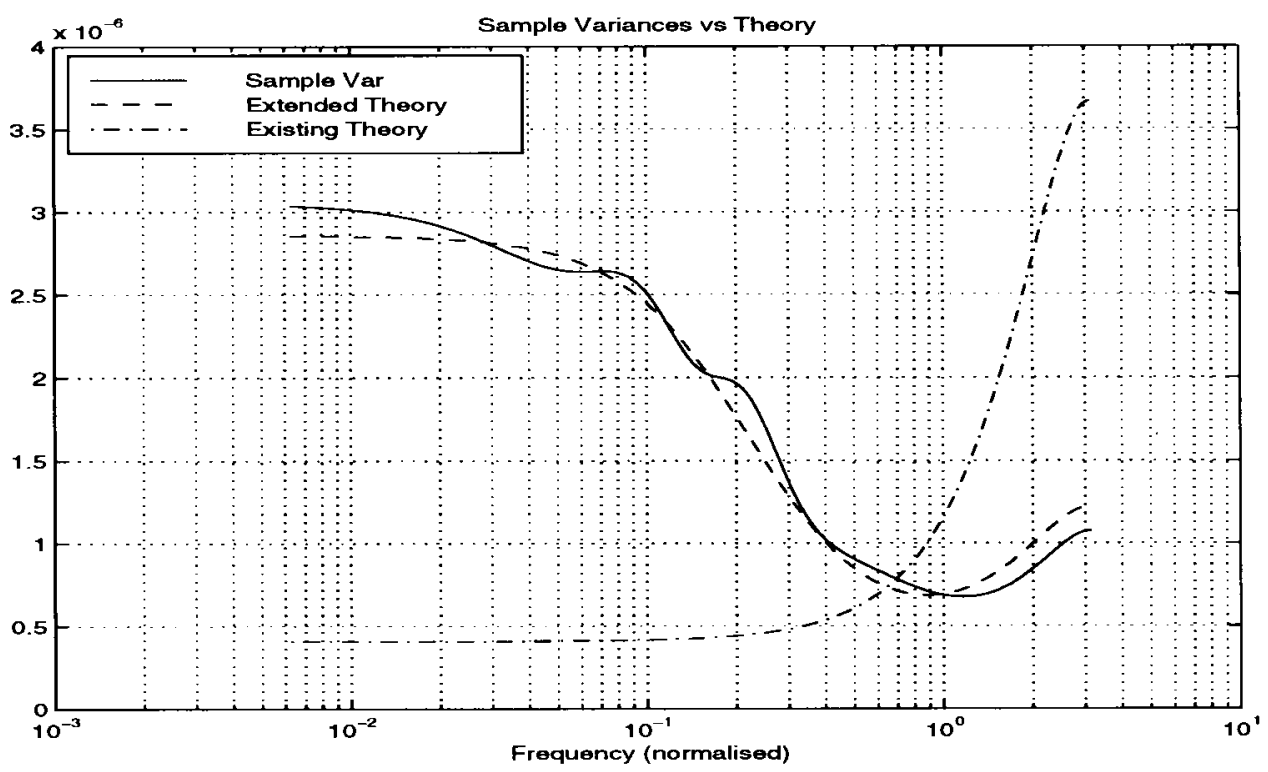

Fig. 3. FIR with eight poles away from origin.

be derived which is shown as the dashed line in Figs. 1-3 and which (by consideration of those figures) can clearly offer greatly improved approximation of the true variability in certain circumstances.

\section{PROBlem Formulation}

Having provided certain motivating arguments, the remainder of the paper proceeds more formally. The problems studied in this paper are ones in which $N$ point data records of an input sequence $\left\{u_{t}\right\}$ and output sequence $\left\{y_{t}\right\}$ of a linear time invariant system are available. It is assumed that this data is generated as follows:

$$
y_{t}=G(q) u_{t}+H(q) e_{t}
$$

Here $G(q)$ is an unknown transfer function describing, in terms of the forward shift operator $q$, the system dynamics that are to be identified by means of the observations $\left\{u_{t}\right\}$ and $\left\{y_{t}\right\}$. The output measurements $\left\{y_{t}\right\}$ are corrupted by a zero mean and stationary noise sequence $\nu_{t}=H(q) e_{t}$ where $H(q)$ is a stable and stably invertible and monic transfer function and $\left\{e_{t}\right\}$ is a zero mean white noise sequence with variance $\mathbf{E}\left\{e_{t}^{2}\right\}=\sigma^{2}<\infty$ and with $\mathbf{E}\left\{\left|e_{t}\right|^{4+\delta}\right\}<\infty$ for some $\delta>0$. The input $\left\{u_{t}\right\}$ is assumed to be quasi-stationary in the sense used by Ljung [20] (or, equivalently, to be amenable to the "generalized Harmonic analysis" of Wiener [48]) so that it has an associated spectral density $\Phi_{u}(\omega)>0$ which is assumed to be Lipschitz continuous of some order $\epsilon>0$. The spectral density of the noise process $\left\{\nu_{t}\right\}$ is denoted as $\Phi_{\nu}(\omega)=\sigma^{2}\left|H\left(e^{j \omega}\right)\right|^{2}$ and is also assumed to be positive and Lipschitz continuous of order $\epsilon>0$.

The identification schemes considered here are ones in which the prediction error framework [20], [43], [5] is used. This requires a model structure

$$
y_{t}=G\left(q, \theta^{n}\right) u_{t}+H\left(q, \theta^{n}\right) e_{t}
$$

parameterized by a vector $\theta^{n} \in \boldsymbol{R}^{n}$ to be employed which is of the form

$G\left(q, \theta^{n}\right)=\sum_{k=1}^{\infty} g_{k}\left(\theta^{n}\right) q^{-k}, \quad H\left(q, \theta^{n}\right)=1+\sum_{k=1}^{\infty} h_{k}\left(\theta^{n}\right) q^{-k}$

This structure implies the following one-step-ahead predictor:

$$
\hat{y}_{t}\left(\theta^{n}\right)=\left[1-H^{-1}\left(q, \theta^{n}\right)\right] y_{t}+H^{-1}\left(q, \theta^{n}\right) G\left(q, \theta^{n}\right) u_{t}
$$

and associated prediction error

$$
\varepsilon_{t}\left(\theta^{n}\right) \triangleq y_{t}-\hat{y}_{t}\left(\theta^{n}\right)
$$

so that if the quadratic criterion

$$
V_{N}\left(\theta^{n}\right) \triangleq \frac{1}{2 N} \sum_{t=1}^{N} \varepsilon_{t}^{2}\left(\theta^{n}\right)
$$

is employed, then based on the $N$ point data observation, a least squares estimate $\hat{\theta}_{N}^{n}$ of $\theta^{n}$ may be found as

$$
\hat{\theta}_{N}^{n} \triangleq \underset{\theta^{n} \in \boldsymbol{R}^{n}}{\arg \min } V_{N}\left(\theta^{n}\right) .
$$

The theory pertaining to the properties of such a method is very rich. Germane to this paper are the properties that [20], [43], [5]

$$
\hat{\theta}_{N}^{n} \stackrel{\text { a.s. }}{\longrightarrow} \theta_{o}^{n} \text { as } N \rightarrow \infty
$$

where with $\{\mathbf{E} \cdot\}$ denoting expectation over the underlying probability space that any random variables are defined on

$$
\theta_{o}^{n} \triangleq \underset{\theta^{n} \in \boldsymbol{R}^{n}}{\operatorname{argmin}} \lim _{N \rightarrow \infty} \mathbf{E}\left\{V_{N}\left(\theta^{n}\right)\right\} .
$$

As well [23], [20]

$$
\sqrt{N}\left(\hat{\theta}_{N}^{n}-\theta_{o}^{n}\right) \stackrel{\mathcal{D}}{\rightarrow} \mathcal{N}\left(0, P_{n}\right), \text { as } N \rightarrow \infty
$$


where

$$
P_{n} \triangleq R_{n}^{-1} Q_{n} R_{n}^{-1}
$$

and with the definition of the prediction error gradient $\psi_{t}\left(\theta^{n}\right)$ as

$$
\psi_{t}\left(\theta^{n}\right)=\frac{d}{d \theta^{n}} \hat{y}_{t}\left(\theta^{n}\right)
$$

then

$$
\begin{aligned}
R_{n} \triangleq & \lim _{N \rightarrow \infty} \mathbf{E}\left\{\left.\frac{d^{2} V_{N}(\theta)}{d \theta \theta^{T}}\right|_{\theta=\theta_{o}^{n}}\right\} \\
= & \lim _{N \rightarrow \infty} \frac{1}{N} \sum_{t=1}^{N} \mathbf{E}\left\{\psi_{t}\left(\theta_{o}^{n}\right) \psi_{t}^{T}\left(\theta_{o}^{n}\right)\right\} \\
& -\lim _{N \rightarrow \infty} \frac{1}{N} \sum_{t=1}^{N} \mathbf{E}\left\{\varepsilon_{t}\left(\theta^{n}\right)\left(\frac{d \psi_{t}\left(\theta^{n}\right)}{d \theta^{n}}\right)^{T}\right\}
\end{aligned}
$$

and

$$
\begin{aligned}
Q_{n} & \triangleq \lim _{N \rightarrow \infty} N \mathbf{E}\left\{\frac{d V_{N}\left(\theta_{o}^{n}\right)}{d \theta^{n}}\left(\frac{d V_{N}\left(\theta_{o}^{n}\right)}{d \theta^{n}}\right)^{T}\right\} \\
& =\lim _{N \rightarrow \infty} \frac{1}{N} \sum_{t=1}^{N} \sum_{\ell=1}^{N} \mathbf{E}\left\{\psi_{t}\left(\theta_{o}^{n}\right) \psi_{\ell}\left(\theta_{o}^{n}\right)^{T} \varepsilon_{t}\left(\theta_{o}^{n}\right) \varepsilon_{\ell}\left(\theta_{o}^{n}\right)\right\} .
\end{aligned}
$$

A key contribution of [20], [22], [25] is to recognize that in applications, often the quantification of the parameter space properties of $\hat{\theta}_{N}^{n}$ are of secondary importance to their influence on the associated properties of $G\left(e^{j \omega}, \hat{\theta}_{N}^{n}\right)$ and $H\left(e^{j \omega}, \hat{\theta}_{N}^{n}\right)$. For the purposes of analyzing the latter, it is expedient to define the composite transfer function

$$
\Pi\left(q, \theta^{n}\right) \triangleq\left[G\left(q, \theta^{n}\right), H\left(q, \theta^{n}\right)\right]
$$

and argue by Taylor expansion that

$$
\begin{aligned}
\tilde{\Pi}_{N}(\omega) & \triangleq \Pi\left(e^{j \omega}, \hat{\theta}_{N}^{n}\right)-\Pi\left(e^{j \omega}, \theta_{o}^{n}\right) \\
& =\left[\frac{d}{d \theta^{n}} \Pi\left(e^{j \omega}, \theta_{o}^{n}\right)\right]^{T}\left(\hat{\theta}_{N}^{n}-\theta_{o}^{n}\right)+o\left(\left\|\hat{\theta}_{N}^{n}-\theta_{o}^{n}\right\|\right)
\end{aligned}
$$

where here $\|\cdot\|$ denotes Euclidean norm so that with the notation $\Pi^{\prime}\left(e^{j \omega}, \theta_{o}^{n}\right) \triangleq d \Pi\left(e^{j \omega}, \theta^{n}\right) / d \theta^{n}$ and with $\cdot^{*}$ denoting "conjugate transpose"

$$
\sqrt{N} \tilde{\Pi}_{N}(\omega) \stackrel{\mathcal{D}}{\rightarrow} \mathcal{N}\left(0, \Lambda_{n}(\omega)\right)
$$

where

$$
\Lambda_{n}(\omega)=\left[\Pi^{\prime}\left(e^{j \omega}, \theta_{o}^{n}\right)\right]^{*} P_{n} \Pi^{\prime}\left(e^{j \omega}, \theta_{o}^{n}\right) .
$$

This suggests the approximation

$$
N \mathrm{E}\left\{\tilde{\Pi}_{N}(\omega) \tilde{\Pi}_{N}^{*}(\omega)\right\} \approx \Lambda_{n}(\omega) .
$$

Unfortunately, the evaluation of $\Lambda_{n}(\omega)$ is always too complicated to be useful. A key contribution of [22], [25] is to observe that in contrast to the intractability of $\Lambda_{n}(\omega)$, the limit

$$
\lim _{N, n \rightarrow \infty} \frac{N}{n} \mathbf{E}\left\{\tilde{\Pi}_{N}(\omega) \tilde{\Pi}_{N}^{*}(\omega)\right\}=\lim _{n \rightarrow \infty} \frac{1}{n} \Lambda_{n}(\omega) \triangleq \Lambda(\omega)
$$

does have a simple and useful form; for example $\Lambda(\omega)=$ $\Phi_{\nu}(\omega) / \Phi_{u}(\omega)$ for FIR model structures [25].

The suggestion of [22], [25] is to then assume that even for finite $N$ and $n$ it can reasonably be expected that $n^{-1} \Lambda_{n}(\omega)$ has approximately converged to the simple form $\Lambda(\omega)$ so that

$$
\mathbf{E}\left\{\tilde{\Pi}_{N}(\omega) \tilde{\Pi}_{N}^{*}(\omega)\right\} \approx \frac{n}{N} \Lambda(\omega)
$$

is a good approximation. The validity of this strategy is argued via numerical example in [22], [25], [20], and indeed it has won widespread acceptance as a tool for analyzing the performance of least squares estimation schemes; see for example [8], [21], [2], [12], and [16].

However, Section II has illustrated what seems to be the unappreciated fact that in certain circumstances the accuracy of (15) is not guaranteed even for what might be considered "large" $N$ or $n$. Instead, it is indicated in Figs. 1-3 that the presence of fixed model structure poles that are away from the origin may seriously degrade the accuracy of the approximation (1). The following section provides a theoretical analysis of this previously unrecognized phenomenon.

\section{Fixed Denominator Model Structures}

The observations of Section II will, in the interests of generality, be addressed by considering the class of so-called "fixed denominator" model structures. This terminology will refer to structures of the form (4) in which the poles of $G\left(q, \theta^{n}\right)$ are prespecified and of which the archetypal example is

$$
G\left(q, \theta^{n}\right) \triangleq D_{n}^{-1}(q) \sum_{k=0}^{n-1} \theta_{k}^{n} q^{k}, \quad H\left(q, \theta^{n}\right)=1
$$

with

$$
D_{n}(q)=\prod_{k=0}^{n-1}\left(q-\xi_{k}\right)
$$

for some user chosen poles $\left\{\xi_{k}\right\} \in D \triangleq\{z \in C:|z|<1\}$ where $\boldsymbol{C}$ denotes the field of complex numbers. Although this is the quintessential form, the results to be presented here will apply to a much more general class of "fixed denominator" model structures (54), (55) considered later.

Specialized "orthonormal" versions of this structure have recently attracted significant research attention [44], [45], [15] where it has been suggested that although the choice $\xi_{k}=0$ in (16) gives the common FIR structure, it is intuitively more reasonable to choose the poles $\left\{\xi_{k}\right\}$ according to prior knowledge so as to be close to the suspected true poles of $G(q)$.

In order to explain why when using these fixed denominator model structures, the prima facie applicable results of [25] lead to such a poor approximation illustrated in Fig. 3, it is necessary to precisely examine the steps used in [25] to arrive at (1). For this purpose, first note that in the fixed denominator case the prediction error gradient $\psi_{t}$ is given by

$$
\psi_{t}\left(\theta_{o}^{n}\right)=\Gamma_{n}(q) D_{n}^{-1}(q) u_{t}=\Gamma_{n}(q) \tilde{u}_{t}
$$


where $\tilde{u}_{t} \triangleq D_{n}^{-1}(q) u_{t}$ and

$$
\Gamma_{n}(q) \triangleq\left[1, q, q^{2}, \cdots, q^{n-1}\right]^{T}
$$

Therefore, $d \psi_{t} / d \theta^{n}=0$ so that from (10)

$$
R_{n}=\lim _{N \rightarrow \infty} \frac{1}{N} \sum_{t=1}^{N} \mathbf{E}\left\{\psi_{t}\left(\theta_{o}^{n}\right) \psi_{t}^{T}\left(\theta_{o}^{n}\right)\right\} .
$$

Defining for any positive function $f:[-\pi, \pi] \rightarrow(0, \infty)$ the $n \times n$ symmetric Toeplitz matrix $T_{n}(f)$ by (sometimes, in the interests of readability, $\omega$ will be used in place of $e^{j \omega}$ )

$$
T_{n}(f) \triangleq \frac{1}{2 \pi} \int_{-\pi}^{\pi} \Gamma_{n}(\omega) \Gamma_{n}^{*}(\omega) f(\omega) d \omega
$$

then using Parseval's formula the matrix $R_{n}$, may be written as [20]

$$
R_{n}=T_{n}\left(\Phi_{\tilde{u}}\right)
$$

where clearly $\Phi_{\tilde{u}}=\left|D_{n}\left(e^{j \omega}\right)\right|^{-2} \Phi_{u}(\omega)$. It is also possible [22], for the case of $H(q)=1$ to establish that provided the true system is in the model set then $Q_{n}=\sigma^{2} R_{n}$ so that $P_{n}=\sigma^{2} R_{n}^{-1}=\sigma^{2} T_{n}^{-1}\left(\Phi_{\tilde{u}}\right)$. Furthermore, by employing well-known results on the asymptotic behavior of Toeplitz matrices [11], [47] it is also true that

$$
T_{n}^{-1}\left(\Phi_{\tilde{u}}\right) \approx T_{n}\left(\frac{1}{\Phi_{\tilde{u}}}\right)
$$

where the precise meaning of the $\approx$ operator is that equality occurs in Hilbert-Schmidt weak matrix norm $|\cdot|$ (defined by $\left|A_{n}\right|^{2}=n^{-1}$ Trace $\left.\left\{A_{n}^{T} A_{n}\right\}\right)$ as $n \rightarrow \infty$.

Therefore, since in the fixed denominator case $G\left(q, \theta^{n}\right)=$ $D_{n}^{-1}(q) \Gamma_{n}^{T}(q) \theta^{n}$ and $H\left(q, \theta^{n}\right)=1$ so that $\Pi\left(q, \theta^{n}\right)=$ $G\left(q, \theta^{n}\right)$ and hence $\Pi^{\prime}\left(q, \theta^{n}\right)=D_{n}^{-1}(q) \Gamma_{n}(q)$ then from (14) and the preceding Toeplitz matrix approximation

$$
\Lambda_{n}(\omega) \approx \frac{\sigma^{2}}{\left|D_{n}\left(e^{j \omega}\right)\right|^{2}} \Gamma_{n}^{*}(\omega) T_{n}\left(\frac{1}{\Phi_{\tilde{u}}}\right) \Gamma_{n}(\omega) .
$$

The final key ingredient facilitating the analysis in [25] is to recognize that

$$
\frac{1}{n} \Gamma_{n}^{*}(\omega) T_{n}(f) \Gamma_{n}(\omega)=\sum_{k=-n}^{n}\left(1-\frac{|k|}{n}\right) c_{k} e^{j \omega k}
$$

where the $\left\{c_{k}\right\}$ are the Fourier coefficients of $f(\omega)$ defined by

$$
c_{k} \triangleq \frac{1}{2 \pi} \int_{-\pi}^{\pi} f(\omega) e^{-j \omega k} d \omega
$$

so that the left-hand side of (23) is in fact a Cesàro mean Fourier reconstruction of $f$ which is known [51], provided $f$ is continuous, to converge uniformly to $f$ on its domain and with increasing $n$.
The reasoning underlying the proofs of [25] for the special FIR case of $\left|D_{n}\left(e^{j \omega}\right)\right|=\left|e^{j \omega n}\right|=1$ then is that since by (23) the expression (22) is the $n$ th-order Cesàro mean Fourier reconstruction of $1 / \Phi_{\tilde{u}}(\omega)$, then for large $n$ (22) should be approximately described by

$$
\begin{aligned}
\frac{N}{n} \operatorname{Var}\left\{G\left(e^{j \omega}, \hat{\theta}_{N}^{n}\right)\right\} & \approx \frac{1}{n} \Lambda_{n}(\omega) \\
& \approx \frac{\sigma^{2}}{\left|D_{n}\left(e^{j \omega}\right)\right|^{2}} \frac{1}{\Phi_{\tilde{u}}(\omega)} \\
& =\frac{\sigma^{2}}{\Phi_{u}(\omega)}
\end{aligned}
$$

which is (1).

Now the mechanism leading to the possible inaccuracy of (1) is exposed. In order for (1) to be an accurate approximation, it is necessary that the $n$ th-order Cesàro mean Fourier reconstruction of $1 / \Phi_{\tilde{u}}(\omega)$ inherent in (22) has approximately converged. The size of $n$ for which this may be expected to hold depends [51] on the smoothness of $\Phi_{\tilde{u}}^{-1}(\omega)=$ $\left|D_{n}\left(e^{j \omega}\right)\right|^{2} / \Phi_{u}(\omega)$. For the special FIR case of $\left|D_{n}\left(e^{j \omega}\right)\right|=$ 1 , the smoothness is fixed as the smoothness of $\Phi_{u}(\omega)$ so that the approximation can be expected to monotonically improve with increasing $n$. However, if poles are not chosen at the origin then the smoothness of $\left|D_{n}\left(e^{j \omega}\right)\right|^{2} / \Phi_{u}(\omega)$ may decrease with increasing $n$ so that convergence of (22) with increasing $n$ need not occur.

Put another way, for poles of $D_{n}(q)$ not at the origin, the Fourier reconstruction inherent in (22) is with respect to coefficients which are more accurately labeled as $\left\{c_{k}^{n}\right\}$ rather than $\left\{c_{k}\right\}$ since they depend on the model order $n$ because they are the Fourier coefficients of $\left|D_{n}\right|^{2} / \Phi_{u}$. In this case, as the length $n$ of the Fourier reconstruction grows, the coefficients may change if $\left|D_{n}\left(e^{j \omega}\right)\right|$ changes, so there is no guarantee that approximate convergence has occurred for "large" but finite $n$.

Therefore, the more poles that are chosen that are away from the origin, and hence the more dependent on $n$ that $\left|D_{n}\left(e^{j \omega}\right)\right|$ is, the less one should rely on (1) applying for finite $n$ since the less likely it is that the underlying Fourier series has come close to convergence.

This is precisely the behavior demonstrated in Figs. 1-3 of Section II.

\section{VARIANCE ERROR FOR FIXED DENOMINATOR MODEL STRUCTURE}

Initially, this analysis of the utility of (1) for certain model structures may seem pessimistic since it indicates that much higher model orders $n$ will be necessary before (1) can be used. A main contribution of this paper is to show that in fact this pessimism is unfounded since, as precursed by the dashed lines of Figs. 2 and 3, it is possible to derive improved approximations that do not require increased model complexity in order to be accurate.

Central to achieving this goal is the consideration of particular rational basis functions, hereafter denoted as the set $\left\{\mathcal{B}_{k}(q)\right\}$ and defined by (- denotes complex conjugation of 
scalar quantities)

$$
\begin{aligned}
& \mathcal{B}_{0}(q) \triangleq \frac{\sqrt{1-\left|\xi_{0}\right|^{2}}}{q-\xi_{0}} \\
& \mathcal{B}_{k}(q) \triangleq \frac{\sqrt{1-\left|\xi_{k}\right|^{2}}}{q-\xi_{k}} \prod_{m=0}^{k-1}\left(\frac{1-\overline{\xi_{m}} q}{q-\xi_{m}}\right) ; \quad k \geq 1 .
\end{aligned}
$$

They are orthonormal according to the inner product definition

$$
\begin{aligned}
\langle f, g\rangle & \triangleq \frac{1}{2 \pi} \int_{-\pi}^{\pi} f\left(e^{j \omega}\right) \overline{g\left(e^{j \omega}\right)} d \omega \\
& =\frac{1}{2 \pi j} \oint_{\boldsymbol{T}} f(z) \overline{g(z)} \frac{d z}{z}
\end{aligned}
$$

where $\boldsymbol{T} \triangleq\{z \in \boldsymbol{C}:|z|=1\}$; the orthonormality following easily using Cauchy's integral formula. These basis functions have been discussed in detail in [34] in the context of generalizing certain other orthonormal bases existing in the system identification literature [44], [45], [37], [15].

One of the main contributions of this paper is to recognize that the asymptotic frequency domain properties of $G\left(e^{j \omega}, \hat{\theta}_{N}^{n}\right)$ are invariant to reparameterizations of the model structure, and to therefore not attempt the scrutiny of (16) directly, but instead consider its reparameterized form

$$
G\left(q, \theta^{n}\right)=\sum_{k=0}^{n-1} \theta_{k}^{n} \mathcal{B}_{k}(q), \quad H\left(q, \theta^{n}\right)=1
$$

which, as will be demonstrated, is much more amenable to analysis than the original form (16).

Pivotal to the ensuing study employing this idea is the recognition of the importance of the so-called "reproducing kernel" $K_{n}(z, \mu)$ associated with the space $X_{n} \triangleq \operatorname{Span}\left\{\mathcal{B}_{0}, \mathcal{B}_{1}, \cdots, \mathcal{B}_{n-1}\right\}$ and which may be expressed as

$$
K_{n}(z, \mu)=\sum_{k=1}^{n-1} \overline{\mathcal{B}_{k}(\mu)} \mathcal{B}_{k}(z), \quad z, \mu \in \boldsymbol{C} .
$$

It derives its name from the property that for any $f \in X_{n}$ and $\mu \in \boldsymbol{C}$ such that $\mu \notin\left\{\xi_{0}, \cdots, \xi_{n-1}\right\}$ then

$$
f(\mu)=\left\langle f(z), K_{n}(z, \mu)\right\rangle
$$

and by virtue of which $K_{n}(z, \mu)$ is unique [35]. In Section VI it will be shown how the property (29) can be exploited in order to quantify bias error, but to do so a more explicit expression for (28) is required. This has been derived in [35] where in analogy with equivalent formulas in the study of orthogonal polynomials [11], [41] it is termed a "Christoffel-Darboux" formula

$$
K_{n}(z, \mu)=\frac{1-\overline{\varphi_{n}(\mu)} \varphi_{n}(z)}{\bar{\mu} z-1}, \quad z, \mu \in \mathbf{D} .
$$

In this expression, $\varphi_{n}(z)$ is defined as the Blaschke productlike quantity

$$
\varphi_{0}(z)=1, \quad \varphi_{n}(z) \triangleq \prod_{k=0}^{n-1}\left(\frac{1-\overline{\xi_{k}} z}{z-\xi_{k}}\right), \quad n \geq 1 .
$$

However, deferring the question of bias error until later, the purpose of this section is to quantify variance error, and for this purpose it is necessary to modify the original Toeplitz matrix formulation (20) to

$$
M_{n}(f) \triangleq \frac{1}{2 \pi} \int_{-\pi}^{\pi} \Gamma_{n}(\omega) \Gamma_{n}^{*}(\omega) f(\omega) d \omega
$$

which although formally identical to (31), is functionally quite different in that the underlying orthonormal basis is not fixed at the trigonometric one, but a generalization obtained by redefining $\Gamma_{n}(z)$ from (18) to

$$
\Gamma_{n}(z) \triangleq\left[\mathcal{B}_{0}(z), \mathcal{B}_{1}(z), \cdots, \mathcal{B}_{n-1}(z)\right]^{T} .
$$

Matrices defined by (31), (32) will be called "generalized Toeplitz" matrices, with the epithet deriving from the fact that if all the poles $\left\{\xi_{k}\right\}$ are chosen at the origin then $M_{n}(f)=$ $T_{n}(f)$ is a bona-fide Toeplitz matrix, but otherwise it is not.

Pertinent to the employment of the matrices (31) in this paper is that with $\|\cdot\|$ denoting the matrix two-norm [9] and as shown in [35] the following bounds apply:

$$
\left\|M_{n}(f)\right\| \leq\|f\|_{\infty}, \quad\left\|M_{n}^{-1}(f)\right\| \leq\|1 / f\|_{\infty} .
$$

Using the new notation (31), (32) the reparameterized model structure (27) may be written as $G\left(q, \theta^{n}\right)=\Gamma_{n}^{T}(q) \theta^{n}$ so that $\psi_{t}\left(\theta_{o}^{n}\right)=\Gamma_{n}(q)$ and hence using (19) and Parseval's formula $R_{n}$ is a generalized Toeplitz matrix of the form

$$
R_{n}=M_{n}\left(\Phi_{u}\right) .
$$

The matrix $Q_{n}$ is more difficult to handle. To expand on this, note that with the formulation

$$
z_{k}\left(\theta_{o}^{n}\right) \triangleq\left[G(q)-G\left(q, \theta_{o}^{n}\right)\right] u_{k}
$$

then $\varepsilon_{k}\left(\theta_{o}^{n}\right)=z_{k}\left(\theta_{o}^{n}\right)+\nu_{k}$ and hence using the definition (11)

$$
\begin{aligned}
Q_{n}= & \lim _{N \rightarrow \infty} \frac{1}{N} \sum_{k=0}^{N-1} \sum_{m=0}^{N-1} \mathbf{E}\left\{\psi_{k} \psi_{m}^{T}\left[z_{k}\left(\theta_{o}^{n}\right)+\nu_{k}\right]\right. \\
& \left.\cdot\left[z_{m}\left(\theta_{o}^{n}\right)+\nu_{m}\right]\right\} .
\end{aligned}
$$

If the data are collected in open loop so that $\left\{\psi_{k}\right\}$ and $\left\{\nu_{k}\right\}$ are uncorrelated, then this can be rewritten as

$$
\begin{aligned}
Q_{n}= & \lim _{N \rightarrow \infty} \frac{1}{N} \sum_{k=0}^{N-1} \sum_{m=0}^{N-1} \mathbf{E}\left\{\psi_{k} \psi_{m}^{T}\right\} \mathbf{E}\left\{\nu_{k} \nu_{m}\right\} \\
& +\lim _{N \rightarrow \infty} \frac{1}{N} \sum_{k=0}^{N-1} \sum_{m=0}^{N-1} \mathbf{E}\left\{\psi_{k} \psi_{m}^{T} z_{k}\left(\theta_{o}^{n}\right) z_{m}\left(\theta_{o}^{n}\right)\right\} .
\end{aligned}
$$

Considering only the first part of this expression, use of Lemma A.1 in the Appendix permits its expression as

$$
\begin{aligned}
\lim _{N \rightarrow \infty} & \frac{1}{N} \sum_{k=0}^{N-1} \sum_{m=0}^{N-1} \mathbf{E}\left\{\psi_{k} \psi_{m}^{T}\right\} \mathbf{E}\left\{\nu_{k} \nu_{m}\right\} \\
& =\frac{1}{2 \pi} \int_{-\pi}^{\pi} \Gamma_{n}(\omega) \Gamma_{n}^{*}(\omega) \Phi_{u}(\omega) \Phi_{\nu}(\omega) d \omega .
\end{aligned}
$$

This result (37) has been used in previous work [44], [45], [37], [34] related to the results of this section but despite its 
importance, to the knowledge of the authors, a proof of (37) has not previously appeared in any literature.

This still leaves the term containing $\left\{z_{k}\left(\theta_{o}^{n}\right)\right\}$ components to be dealt with. Clearly, from definition (34) these terms are due to undermodeling, and if it so happens that $G(q)$ is in the model set, then they will be zero and hence (37) will completely describe $Q_{n}$. In the seminal work [25], [22], the assumption of $G(q)$ being in the model set for finite $n$ was not made, and instead the effect of the $\left\{z_{k}\left(\theta_{o}^{n}\right)\right\}$ components was directly addressed. In subsequent work [44], [45], [37], [34] related to this paper it has been assumed that since eventually $n \rightarrow \infty$ is to be considered, then the effect of $z_{k}\left(\theta_{o}^{n}\right)$ will eventually be inconsequential and hence can be neglected from the beginning. However, since $z_{k}\left(\theta_{o}^{n}\right)$ enters into the formulation of $\Lambda_{n}(\omega)$ in such a complicated way, it is not obvious that its effect on $\Lambda_{n}(\omega)$ will tend to zero with increasing $n$ even if $z_{k}\left(\theta_{o}^{n}\right)$ itself does.

As a result, this paper elects to in fact account for it by using (37) to write $Q_{n}$ as

$$
Q_{n}=M_{n}\left(\Phi_{u} \Phi_{\nu}\right)+\Delta_{n}
$$

where $\Delta_{n}$ is the second term of (36) which, if neglected $a b$ initio as in [44], [45], [37], implies a presumption of $\lim _{n \rightarrow \infty}\left\|\Delta_{n}\right\|=0$.

With these comments in hand, the analysis can proceed by noting that from (9)

$$
\begin{aligned}
P_{n}= & M_{n}^{-1}\left(\Phi_{u}\right) M_{n}\left(\Phi_{u} \Phi_{\nu}\right) M_{n}^{-1}\left(\Phi_{u}\right) \\
& +M_{n}^{-1}\left(\Phi_{u}\right) \Delta_{n} M_{n}^{-1}\left(\Phi_{u}\right) .
\end{aligned}
$$

With the reparameterization (27), then $G\left(q, \theta^{n}\right)=\Gamma_{n}^{T}(q) \theta^{n}$ with $\Gamma_{n}(q)$ being the generalized form (32) so that $\Pi^{\prime}\left(q, \theta^{n}\right)=$ $\Gamma_{n}(q)$ (the derivative with respect to $H(q)$ has been ignored since it is zero) and hence from (14) the quantity $\Lambda_{n}(\omega)$ quantifying variance error may be written as

$$
\begin{aligned}
\Lambda_{n}(\omega)= & \Gamma_{n}^{*}(\omega) M_{n}^{-1}\left(\Phi_{u}\right) M_{n}\left(\Phi_{u} \Phi_{\nu}\right) M_{n}^{-1}\left(\Phi_{u}\right) \Gamma_{n}(\omega) \\
& +\Gamma_{n}^{*}(\omega) M_{n}^{-1}\left(\Phi_{u}\right) \Delta_{n} M_{n}^{-1}\left(\Phi_{u}\right) \Gamma_{n}(\omega)
\end{aligned}
$$

In order to simplify this for large $n$ it is necessary to know something of the asymptotic algebraic structure of the generalized Toeplitz matrices in this expression, and this turns out to be a somewhat delicate question.

In related work [11], [47] pertaining to "classical" symmetric Toeplitz matrices $T_{n}(f)$, it is shown that $\mid T_{n}(f) T_{n}(g)-$ $T_{n}(f g) \mid \rightarrow 0$ as $n \rightarrow \infty$ where $|\cdot|$ denotes the Hilbert-Schmidt weak matrix norm defined earlier. In the generalized case considered here, an equivalent result cannot be shown to hold without overly restrictive assumptions being imposed [35]. Instead, it is necessary to be more subtle and recognize that in applications what is really important is how products $M_{n}(f) M_{n}(g)$ affect vectors only in the directions specified by $\Gamma_{n}(\omega)$.

This principle leads to the notion of asymptotic equivalence between two $n \times n$ matrices $A_{n}$ and $B_{n}$ with notation

$$
A_{n} \sim B_{n} \quad \text { as } n \rightarrow \infty
$$

if for all $\omega \in[-\pi, \pi]$

$$
\lim _{n \rightarrow \infty} \frac{\Gamma_{n}^{*}(\omega)\left[A_{n}-B_{n}\right]\left[A_{n}-B_{n}\right]^{*} \Gamma_{n}(\omega)}{K_{n}(\omega, \omega)}=0 .
$$

The utility of this idea is that if $A_{n} \sim B_{n}$ as $n \rightarrow \infty$ then the asymptotic consideration of quadratic forms of $A_{n}$ and $B_{n}$ in $\Gamma_{n}(\omega)$ are identical. Specifically, for any $\omega, \lambda \in[-\pi, \pi]$ and using the Cauchy-Schwarz inequality

$$
\begin{aligned}
\left|\frac{\Gamma_{n}^{*}(\omega)}{K_{n}(\omega, \omega)}-\frac{A_{n} \Gamma_{n}(\lambda)}{K_{n}(\omega, \omega)}\right|^{*}(\omega) B_{n} \Gamma_{n}(\lambda) \\
=\left|\frac{\Gamma_{n}^{*}(\omega)\left[A_{n}-B_{n}\right] \Gamma_{n}(\lambda)}{K_{n}(\omega, \omega)}\right|^{2} \\
\leq\left|\frac{\Gamma_{n}^{*}(\omega)\left[A_{n}-B_{n}\right]\left[A_{n}-B_{n}\right]^{*} \Gamma_{n}(\omega)}{K_{n}(\omega, \omega)}\right| \\
\quad \times\left|\frac{\Gamma_{n}^{*}(\lambda) \Gamma_{n}(\lambda)}{K_{n}(\omega, \omega)}\right| .
\end{aligned}
$$

However, taking advantage of the explicit forms of the reproducing kernel made possible by (28) and (25)

$$
\begin{aligned}
\Gamma_{n}^{*}(\lambda) \Gamma_{n}(\lambda)=K_{n}(\lambda, \lambda) & =\sum_{k=0}^{n-1}\left|\mathcal{B}_{k}\left(e^{j \lambda}\right)\right|^{2} \\
& =\sum_{k=0}^{n-1} \frac{1-\left|\xi_{k}\right|^{2}}{\left|e^{j \lambda}-\xi_{k}\right|^{2}} \\
& \leq \mu^{2} \sum_{k=0}^{n-1} \frac{1-\left|\xi_{k}\right|^{2}}{\left|e^{j \omega}-\xi_{k}\right|^{2}} \\
& =\mu^{2} K_{n}(\omega, \omega)
\end{aligned}
$$

where $\mu=\max _{\lambda, k}\left|e^{j \omega}-\xi_{k}\right| /\left|e^{j \lambda}-\xi_{k}\right|$ is some constant independent of $n$ that is guaranteed to be finite provided all the poles $\left\{\xi_{k}\right\}$ are chosen in some closed subset of the open unit disk D. Substituting (43) in (41) then shows that the latter tends to zero with increasing $n$, thereby establishing the asymptotic equivalence of the quadratic forms. Note the key role played by the reproducing kernel $K_{n}(\omega, \omega)$.

Of fundamental importance in using these principles to clarify (38) are the results of [35] showing that if $f$ and $g$ are any (possibly complex-valued) functions that are invertible and Lipschitz continuous of some order $\epsilon>0$, and if all the poles $\left\{\xi_{k}\right\}$ are chosen in some closed subset of the open unit $\operatorname{disc} \mathbf{D}$, then

$$
\begin{aligned}
M_{n}(f) M_{n}(g) & \sim M_{n}(f g) \quad \text { as } n \rightarrow \infty \\
M_{n}^{-1}(f) & \sim M_{n}(1 / f) \quad \text { as } n \rightarrow \infty .
\end{aligned}
$$

Applying these results to (38) then provides the formulation

$$
P_{n} \sim M_{n}\left(\frac{\Phi_{\nu}}{\Phi_{u}}\right)+M_{n}^{-1}\left(\Phi_{u}\right) \Delta_{n} M_{n}^{-1}\left(\Phi_{u}\right)
$$

as $n \rightarrow \infty$ so that using the mechanism illustrated in (41)

$$
\begin{aligned}
\lim _{n \rightarrow \infty} \frac{\Lambda_{n}(\omega)}{K_{n}(\omega, \omega)}= & \lim _{n \rightarrow \infty} \frac{\Gamma_{n}^{*}(\omega) M_{n}\left(\Phi_{\nu} / \Phi_{u}\right) \Gamma_{n}(\omega)}{K_{n}(\omega, \omega)} \\
& +\delta_{n}(\omega)
\end{aligned}
$$


where

$$
\delta_{n}(\omega) \triangleq \frac{\Gamma_{n}^{*}(\omega) M_{n}^{-1}\left(\Phi_{u}\right) \Delta_{n} M_{n}^{-1}\left(\Phi_{u}\right) \Gamma_{n}(\omega)}{K_{n}(\omega, \omega)}
$$

which using (33), the Cauchy-Schwarz inequality, and the explicit form (28), can be bounded as

$$
\left|\delta_{n}(\omega)\right| \leq\left\|1 / \Phi_{u}\right\|_{\infty}^{2}\left\|\Delta_{n}\right\| .
$$

In order to further simplify (47) to some useful form, it is pivotal that a generalized analog of the Fourier convergence of (23) exists. Such a result is provided in [35] where it is shown that provided $\Sigma\left(1-\left|\xi_{k}\right|\right)=\infty$ then for any possibly complex-valued but continuous $f$

$$
\lim _{n \rightarrow \infty} \frac{1}{K_{n}(\omega, \omega)} \Gamma_{n}^{*}(\omega) M_{n}(f) \Gamma_{n}(\lambda)= \begin{cases}f(\omega), & \omega=\lambda \\ 0, & \omega \neq \lambda\end{cases}
$$

Again observe the key role played by the reproducing kernel, and in particular note that use of the formulation (28), (25) with $\xi_{k}=0$ gives $K_{n}(\omega, \omega)=n$ so that the left-hand side of (50) becomes the Cesàro mean (23). Because of this, the result (50) can be seen to expand Fourier convergence analysis to the general orthonormal basis (25) which contains the classical trigonometric basis of (23) as a special case of $\xi_{k}=0$. In fact, (50) is something more than this, since it also shows convergence to zero if $\lambda \neq \omega$. In the special trigonometric case this latter specialization appears to have been first established in [13].

Applying (50) to (47) then provides the result that (assume

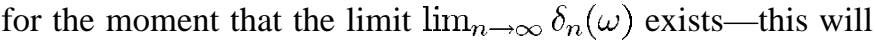
be verified later)

$$
\lim _{n \rightarrow \infty} \frac{\Lambda_{n}(\omega)}{K_{n}(\omega, \omega)}=\frac{\Phi_{\nu}(\omega)}{\Phi_{u}(\omega)}+\lim _{n \rightarrow \infty} \delta_{n}(\omega) .
$$

The dividend of considering the orthonormal reparameterization (27) rather than the more natural fixed denominator form (16) is now revealed. Namely, in doing so (51) is derived from asymptotic analysis of an expression (47) which involves generalized Fourier analysis of an underlying function $\Phi_{\nu} / \Phi_{u}$ whose smoothness is invariant to model order or fixed pole selection. This crucial feature will permit the derivation of the improved variance error approximation shown in Figs. 1-3.

Before further elucidating this point, the developments of this section are formally collected in the following theorem and corollary.

Theorem V.1: With $\hat{\theta}_{N}^{n}$ calculated via (7) using the model structures (16), (27) or any other structure with the same fixed poles $\left\{\xi_{k}\right\}$ all chosen to lie within the open unit disk $\mathbf{D}$, then with $K_{n}(\omega, \omega) \equiv K_{n}\left(e^{j \omega}, e^{j \omega}\right)$ given by (28) and in the limit as $N \rightarrow \infty$

$$
\begin{aligned}
& \sqrt{N}\left[\begin{array}{cc}
K_{n}(\omega, \omega) & 0 \\
0 & K_{n}(\lambda, \lambda)
\end{array}\right]^{-1 / 2}\left(\begin{array}{l}
G\left(e^{j \omega}, \hat{\theta}_{N}^{n}\right)-G\left(e^{j \omega}, \theta_{o}^{n}\right) \\
G\left(e^{j \lambda}, \hat{\theta}_{N}^{n}\right)-G\left(e^{j \lambda}, \theta_{o}^{n}\right)
\end{array}\right) \\
& \quad \stackrel{\mathcal{D}}{\rightarrow} \mathcal{N}\left(0, \Sigma_{n}(\omega, \lambda)\right)
\end{aligned}
$$

where, provided the data is collected in open loop, all the poles $\left\{\xi_{k}\right\}$ are chosen in a closed subset of D and $\Phi_{u}(\omega), \Phi_{\nu}(\omega)$ are Lipschitz continuous of some order $\epsilon>0$, then with the definition

$$
\delta(\omega) \triangleq \lim _{n \rightarrow \infty} \delta_{n}(\omega)
$$

where $\delta_{n}(\omega)$ is defined by (48), then

$$
\lim _{n \rightarrow \infty} \Sigma_{n}(\omega, \lambda)=\left[\begin{array}{cc}
\frac{\Phi_{\nu}(\omega)}{\Phi_{u}(\omega)} & \tilde{\delta}(\omega) \\
\tilde{\delta}(\omega) & \frac{\Phi_{\nu}(\lambda)}{\Phi_{u}(\lambda)}
\end{array}\right]+\delta(\omega) I
$$

where for some finite $\mu$

$$
|\tilde{\delta}(\omega)| \leq \mu|\delta(\omega)| .
$$

Proof: It has already been established in (13) and (14) that

$$
\begin{aligned}
& \sqrt{N}\left[G\left(e^{j \omega}, \hat{\theta}_{N}^{n}\right)-G\left(e^{j \omega}, \hat{\theta}_{o}^{n}\right)\right] \\
& \quad \stackrel{\mathcal{D}}{\rightarrow} \mathcal{N}\left(0, \Lambda_{n}(\omega)\right) \quad \text { as } N \rightarrow \infty
\end{aligned}
$$

where $\Lambda_{n}(\omega)=\Gamma_{n}^{*}(\omega) P_{n} \Gamma_{n}(\omega)$. An extension of this is that as $N \rightarrow \infty$

$$
\sqrt{N}\left[\begin{array}{l}
G\left(e^{j \omega}, \hat{\theta}_{N}^{n}\right)-G\left(e^{j \omega}, \theta_{o}^{n}\right) \\
G\left(e^{j \lambda}, \hat{\theta}_{N}^{n}\right)-G\left(e^{j \lambda}, \theta_{o}^{n}\right)
\end{array}\right] \stackrel{\mathcal{D}}{\rightarrow} \mathcal{N}\left(0, \tilde{\Sigma}_{n}(\omega, \lambda)\right)
$$

where

$$
\tilde{\Sigma}_{n}(\omega, \lambda) \triangleq\left[\begin{array}{ll}
\Gamma_{n}^{*}(\omega) P_{n} \Gamma_{n}(\omega) & \Gamma_{n}^{*}(\omega) P_{n} \Gamma_{n}(\lambda) \\
\Gamma_{n}^{*}(\lambda) P_{n} \Gamma_{n}(\omega) & \Gamma_{n}^{*}(\lambda) P_{n} \Gamma_{n}(\lambda)
\end{array}\right]
$$

so that using the notation of (13), (14)

$$
\begin{aligned}
\sqrt{N} & {\left[\begin{array}{cc}
K_{n}(\omega, \omega) & 0 \\
0 & K_{n}(\lambda, \lambda)
\end{array}\right]^{-1 / 2} } \\
& \cdot\left(\begin{array}{l}
G\left(e^{j \omega}, \hat{\theta}_{N}^{n}\right)-G\left(e^{j \omega}, \theta_{o}^{n}\right) \\
G\left(e^{j \lambda}, \hat{\theta}_{N}^{n}\right)-G\left(e^{j \lambda}, \theta_{o}^{n}\right)
\end{array}\right) \\
& \stackrel{\mathcal{D}}{\rightarrow} \mathcal{N}\left(0, \Sigma_{n}(\omega, \lambda)\right)
\end{aligned}
$$

where

$$
\begin{aligned}
\Sigma_{n}(\omega, \lambda) & \\
= & {\left[\begin{array}{cc}
K_{n}(\omega, \omega) & 0 \\
0 & K_{n}(\lambda, \lambda)
\end{array}\right]^{-1 / 2} \tilde{\Sigma}_{n}(\omega, \lambda) } \\
& \times\left[\begin{array}{cc}
K_{n}(\omega, \omega) & 0 \\
0 & K_{n}(\lambda, \lambda)
\end{array}\right]^{-1 / 2} \\
= & {\left[\begin{array}{cc}
\frac{\Lambda_{n}(\omega)}{K_{n}(\omega, \omega)} & \frac{\Gamma_{n}^{*}(\omega) P_{n} \Gamma_{n}(\lambda)}{\sqrt{K_{n}(\omega, \omega) K_{n}(\lambda, \lambda)}} \\
\frac{\Gamma_{n}^{*}(\lambda) P_{n} \Gamma_{n}(\omega)}{\sqrt{K_{n}(\omega, \omega) K_{n}(\lambda, \lambda)}} & \frac{\Lambda_{n}(\lambda)}{K_{n}(\lambda, \lambda)}
\end{array}\right] . }
\end{aligned}
$$

It has just been established in (51) that

$$
\lim _{n \rightarrow \infty} \frac{\Lambda_{n}(\omega)}{K_{n}(\omega, \omega)}=\lim _{n \rightarrow \infty} \delta_{n}(\omega)+\frac{\Phi_{\nu}(\omega)}{\Phi_{u}(\omega)}
$$

which accounts for the asymptotic value of the diagonal terms of $\Sigma_{n}(\omega, \lambda)$. For the off-diagonal terms note that using the 
formulation (46) and the definition (48) we have

$$
\begin{aligned}
\tilde{\delta}(\omega)= & \frac{\Gamma_{n}^{*}(\omega) P_{n} \Gamma_{n}(\lambda)}{\sqrt{K_{n}(\omega, \omega) K_{n}(\lambda, \lambda)}} \\
= & \frac{\Gamma_{n}^{*}(\omega) M_{n}\left(\Phi_{\nu} / \Phi_{u}\right) \Gamma_{n}(\lambda)}{\sqrt{K_{n}(\omega, \omega) K_{n}(\lambda, \lambda)}} \\
& +\frac{K_{n}(\omega, \omega) \delta_{n}(\omega)}{\sqrt{K_{n}(\omega, \omega) K_{n}(\lambda, \lambda)}} .
\end{aligned}
$$

However, by the argument leading to $(43), K_{n}(\lambda, \lambda) \geq$ $\mu^{2} K_{n}(\omega, \omega)$ for some finite $\mu$ so that

$$
\tilde{\delta}(\omega) \leq \mu \frac{\Gamma_{n}^{*}(\omega) P_{n} \Gamma_{n}(\lambda)}{K_{n}(\omega, \omega)}+\mu \delta(\omega)
$$

Use of (50) then completes the proof.

The main use of this result will be to infer the nature of the variability of $G\left(e^{j \omega}, \hat{\theta}_{N}^{n}\right)$. However, as pointed out in [20], convergence in distribution guarantees nothing about meansquare convergence. Therefore, to be rigorous, it is necessary to separately consider this, but fortunately such examination is straightforward provided the assumptions on the noise process $\left\{e_{t}\right\}$ are somewhat strengthened.

Corollary V.1: Under the same conditions as the previous theorem, but with a strengthened requirement that $\mathbf{E}\left\{e_{t}^{8}\right\}<\infty$, then

$$
\begin{aligned}
\lim _{n \rightarrow \infty} & \lim _{N \rightarrow \infty} \frac{N}{K_{n}(\omega, \omega)} \mathbf{E}\left\{\left|G\left(e^{j \omega}\right)-G\left(e^{j \omega}, \theta_{o}^{n}\right)\right|^{2}\right\} \\
= & \frac{\Phi_{\nu}(\omega)}{\Phi_{u}(\omega)}+\delta(\omega)
\end{aligned}
$$

where

$$
\delta(\omega) \triangleq \lim _{n \rightarrow \infty} \delta_{n}(\omega)
$$

with $\delta_{n}(\omega)$ defined by (48).

Proof: The proof follows from the previous theorem using the methods in [20, Appendix 9B].

By drawing on the precedent of [25], [22] in which the approximation (1) is derived from the asymptotic result (2), this paper uses the new asymptotic result (52) and the explicit formulas (28), (25) to suggest the approximation

$$
\operatorname{Var}\left\{G\left(e^{j \omega}, \hat{\theta}_{N}^{n}\right)\right\} \approx \frac{1}{N} \frac{\Phi_{\nu}(\omega)}{\Phi_{u}(\omega)} \sum_{k=0}^{n-1}\left|\mathcal{B}_{k}\left(e^{j \omega}\right)\right|^{2} .
$$

Note that in providing this approximation, it has been assumed as in [44], [45], and [37] that the undermodeling-induced component $\delta(\omega)$ is negligible, and this simplification will be commented upon later. However, before doing so, there are several important points pertaining to the approximation (53) that need to be highlighted.

Firstly, the expression (53) is shown as a dashed line in the Figs. 1-3 of Section II which, being so close to the sample variability estimate of the true variability, illustrates that in situations where some fixed denominator poles are chosen away from the origin, the new expression (53) can represent an approximation that is significantly more accurate than the well-known one (1). As already suggested, the reason for the improved approximation rests solely with the strategy of reparameterizing with respect to the orthonormal bases (25) in that by doing so, the asymptotic result (52) involves considering convergence of the generalized Fourier expansion (47) of a function $\Phi_{\nu} / \Phi_{u}$ whose smoothness is invariant to the model order, so that the degree of approximation in concluding (53) on the basis of (52) can be expected to monotonically improve with increasing model order $n$.

By using this novel reparameterization approach, the essential feature imbuing the new expression (53) with greater accuracy than the pre-existing approximation (1) is that the influence of the fixed pole location on $\operatorname{Var}\left\{G\left(e^{j \omega}, \hat{\theta}_{N}^{n}\right)\right\}$ is quantified by the reproducing kernel $K_{n}(\omega, \omega)$; see for example Fig. 4 where the expression $K_{n}(\omega, \omega)$ is plotted for a variety of choices of $\left\{\xi_{k}\right\}$. In particular, note that for all poles fixed at the origin, by the formulation (28), (25) then $K_{n}(\omega, \omega)=n$ so that in this special case of FIR modeling, (53) is identical to (1). However, the more poles that are not fixed at the origin, the more $K_{n}(\omega, \omega)$ will (being then frequency dependent) differ from $n$ and hence the more the new approximation (53) will, in the interests of improved accuracy, be perturbed from the original approximation (1).

A point to note is that Fig. 4 indicates that the choice of poles $\left\{\xi_{k}\right\}$ could be conceived as a design variable for decreasing variance error at particular frequencies. However, not only might this incur an increased bias error if the $\left\{\xi_{k}\right\}$ are chosen far from the true poles (see the following section), but Fig. 4 also indicates that a decrease in variance error at one frequency is traded against increases at another frequency. In fact, it is a trivial consequence of orthonormality that the areas (on a linear axis and divided by $2 \pi$ ) underneath all the curves in Fig. 4 are the same and equal to the model order $n$.

Another issue worth emphasizing is that the results in Theorem V.1 and Corollary V.1 and hence in the approximation (53) apply for any model structure (of which (16) and (27) are special cases) of the form

$$
G\left(q, \theta^{n}\right) u_{t}=\phi_{t}^{T} \theta^{n}
$$

where

$$
\phi_{t+1}=A \phi_{t}+B u_{t}
$$

with $A \in \boldsymbol{R}^{n \times n}$ and $B \in \boldsymbol{R}^{n \times 1}$ arbitrary but such that the eigenvalues of $A$ are $\left\{\xi_{0}, \xi_{1}, \cdots, \xi_{n-1}\right\}$.

It seems quite unexpected that this variance quantification role for the orthonormal bases $\left\{\mathcal{B}_{k}\right\}$ given by (25) should arise in such a fundamental manner in a problem that can via (54) and (55) be ab initio formulated with no orthonormality in its structure.

The role of orthonormal bases in system identification is therefore much deeper and more intrinsic than had previously been thought. This provides further motivation for their employment and study that is in addition to the numerical efficiency and modeling efficiency features exposed in previous works [44], [37], [15], [34].

Indeed, previous work which has pioneered the use of orthonormal bases for system identification [44], [45], [37] has also considered variance expressions which are restricted versions of the form (53). For example, in [44] the use 


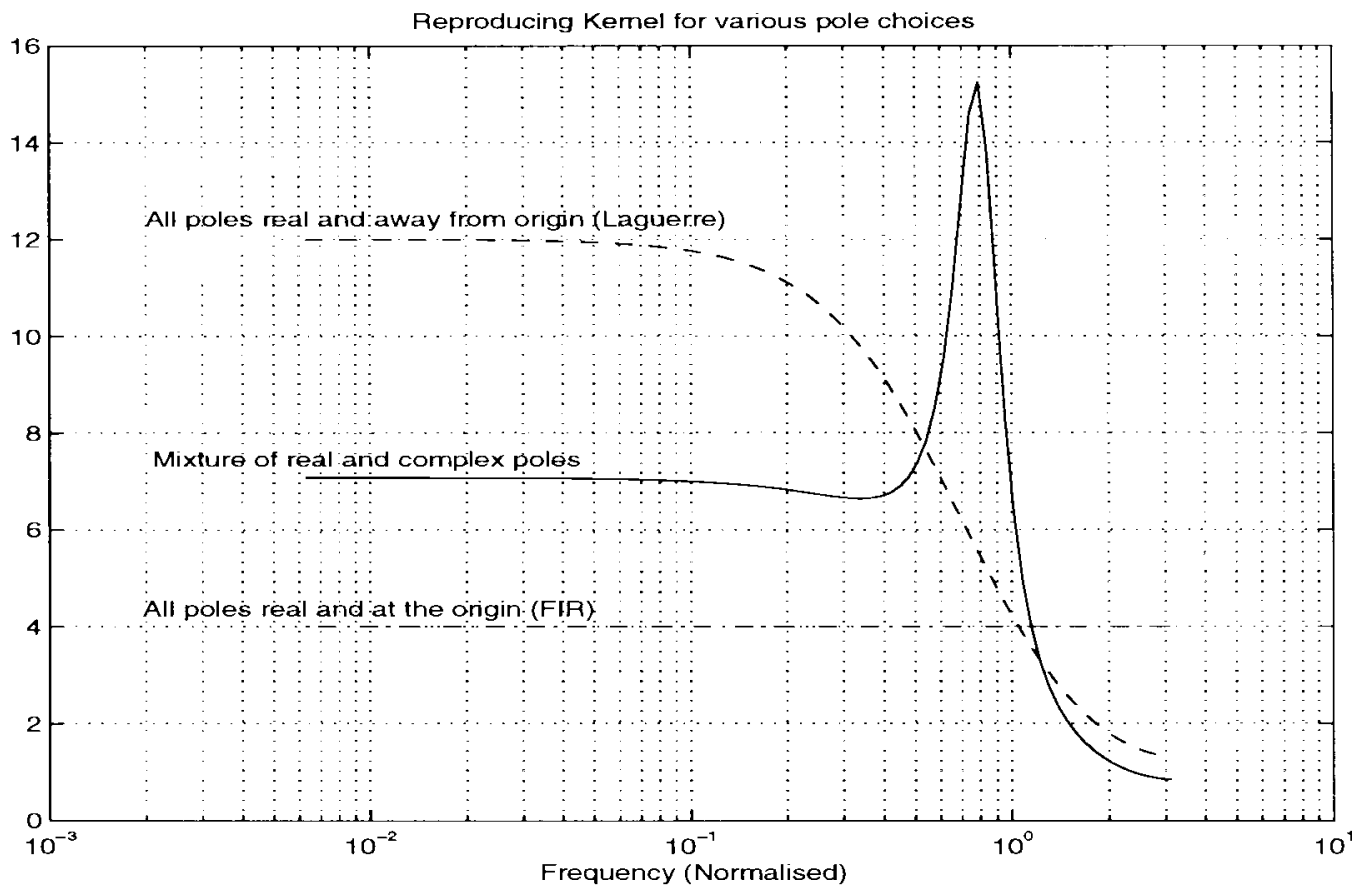

Fig. 4. Plot, for various choices of $\left\{\xi_{k}\right\}$, of term $K_{n}(\omega, \omega)=\Sigma_{k=0}^{n-1}\left|\mathcal{B}_{k}\left(e^{j \omega}\right)\right|^{2}$, which captures the effect of pole choice $\left\{\xi_{k}\right\}$ on the transfer function estimate sensitivity to measurement noise. Here $n=4$.

of Laguerre bases is analyzed. However, this case may be encompassed in the framework of this paper by choosing all the poles the same and real-valued as $\xi_{k}=\xi \in \boldsymbol{R}$ in which case (53) reduces to

$$
\operatorname{Var}\left\{G\left(e^{j \omega}, \hat{\theta}_{N}^{n}\right)\right\} \approx \frac{n}{N} \frac{\Phi_{\nu}(\omega)}{\Phi_{u}(\omega)} \frac{1-\xi^{2}}{\left|e^{j \omega}-\xi\right|^{2}}
$$

which is the same as the result obtained in [44].

In [37], [15], which is the first work to consider the case of possibly different poles, they are still all restricted to be cyclically repeated from a finite set, say $\xi_{k} \in\left\{\xi_{0}, \cdots, \xi_{p-1}\right\}$ and the result

$$
\lim _{m, N \rightarrow \infty} \frac{N}{m \tilde{K}_{p}(\omega, \omega)} \operatorname{Var}\left\{G\left(e^{j \omega}, \hat{\theta}_{N}^{n}\right)\right\}=\frac{\Phi_{\nu}(\omega)}{\Phi_{u}(\omega)}
$$

is derived where $\tilde{K}_{p}(\omega, \omega)=\sum_{k=0}^{p-1}\left|\tilde{\mathcal{B}}_{k}\left(e^{j \omega}\right)\right|^{2}$ with $\left\{\tilde{\mathcal{B}}_{k}\right\}$ being the particular set of orthonormal bases considered in [37]. The quantity $m$ is the number of complete repetitions of the whole set of bases $\left\{\tilde{\mathcal{B}}_{0}, \cdots, \tilde{\mathcal{B}}_{p-1}\right\}$ in the model structure.

This latter point on complete repetitions of pole sets is of crucial importance since it has been demonstrated here that when inferring an approximation like (53) from an asymptotic result like (57) it is vital that convergence of a result like (57) can be reasonably expected to have occurred for finite $m$. However, if all the fixed poles in a model are chosen differently as in Section II, then $m=1$. Therefore, since (57) is asymptotic in $m$, it cannot be argued that (57) can reasonably be expected to have converged in this case of $m=1$, and hence the theoretical justification for an approximation like (53), which is derived on the basis of [37, eq. (57)], is in these cases problematic.

On the other hand, using the methods of this paper, attention is focussed on the asymptotic nature of (47) which converges with increasing model order rather than pole set repetition count. In view of this (and with the precedent of the foundation work [44], [45], [37], [15] in mind) the new techniques proposed here are considered to provide a more theoretically sound argument for inferring a fixed order approximation from an asymptotic result.

Indeed, because of the fixed pole restrictions involved in previous work, the connections made here to general fixed denominator models of the form (54), (55) were never made and hence the fundamental relationship of orthonormal bases to situations which are not $a b$ initio formulated in terms of them has not been previously exposed.

A consequence of the pole restrictions imposed in the previous work [44], [45], [37] is that it forces the bases $\left\{\tilde{\mathcal{B}}_{n}\right\}$ employed there to mimic the algebraic property $e^{j \omega n} e^{j \omega m}=$ $e^{j \omega(m+n)}$ of the classical trigonometric basis. This allows an elegant path to variance results to be followed, wherein the problem is reduced to an equivalent FIR problem by establishing, via a bilinear or multilinear ("Hambo") transform, an algebra isomorphism between $\left\{\tilde{\mathcal{B}}_{n}\right\}$ and $e^{j \omega n}$. The original results of [25] are then mapped through this same isomorphism in order to generalize them [44], [45], [37].

As is evident, the strategy of this paper is completely different and, apart from avoiding pole restrictions, it has the dividend of illustrating new analysis methods employing recently derived results [35] pertaining to generalized Toeplitz matrices and generalized Fourier analysis. These new techniques are considered to be of interest in their own right, as they would appear to have potential for application beyond the particular system identification setting considered here.

The final advantage of the methods pursued here are that they permit an explicit formulation (28), (25) for the term 
$K_{n}(\omega, \omega)$ revealing how fixed poles affect variability; in previous work it has been implicit in a state-space construction [37]. Indeed, by the recognition here that this term $K_{n}(\omega, \omega)$ affecting variability is a reproducing kernel, and hence unique, a closed form expression for the equivalent term in [37] is provided for the first time.

It remains to comment on the undermodeling-induced $\delta_{n}(\omega)$ term in the derivation of (53). Firstly, note that with its omission $\tilde{\delta}(\omega)=0$ in Theorem V.1 so that the frequency response estimates at different frequencies are asymptotically uncorrelated. Note also, that it can be argued that disregarding $\delta_{n}(\omega)$ is reasonable, since if one assumes for the sake of simplicity that $\mathbf{E}\left\{\psi_{k} \psi_{m}^{T} z_{k}\left(\theta_{o}^{n}\right) z_{m}\left(\theta_{o}^{n}\right)\right\}=\mathbf{E}\left\{\psi_{k} \psi_{m}^{T}\right\} \mathbf{E}\left\{z_{k}\left(\theta_{o}^{n}\right) z_{m}\left(\theta_{o}^{n}\right)\right\}$ then using Lemma A.1 and assuming $\left\{z_{k}\left(\theta_{o}^{n}\right)\right\}$ is a stationary stochastic process

$$
\Delta_{n}=M_{n}\left(\left|G-G\left(\theta_{o}^{n}\right)\right|^{2} \Phi_{u}^{2}\right)
$$

so that use of (33) provides

$$
\left\|\Delta_{n}\right\|_{2} \leq\left\|G\left(e^{j \omega}\right)-G\left(e^{j \omega}, \theta_{o}^{n}\right)\right\|_{\infty}\left\|\Phi_{u}(\omega)\right\|_{\infty}^{2} .
$$

In this case, using (49)

$$
\left|\delta_{n}(\omega)\right| \leq\left\|G\left(e^{j \omega}\right)-G\left(e^{j \omega}, \theta_{o}^{n}\right)\right\|_{\infty}\left\|\Phi_{u}\right\|_{\infty}\left\|1 / \Phi_{u}\right\|_{\infty} .
$$

Since it will be proved in Lemma VI.1 that the right-hand side of this inequality tends to zero, theoretical justification which has not been provided in some previous works of neglecting the $\delta_{n}(\omega)$ term is provided.

In fact, this can be made more rigorous in such a way to even address the closed-loop case, but at the cost of more restrictive assumptions.

Theorem V.2: Under the restriction that both $\left\{u_{t}\right\}$ and $\left\{e_{t}\right\}$ are Gaussian-distributed zero mean stationary stochastic processes, then the results of Theorem V.1 and Corollary V.1 hold even for closed-loop data collection and with the $\delta(\omega)$ term guaranteed to be identically zero provided that the substitution

$$
\Phi_{\nu}(\omega) \mapsto \Phi_{\varepsilon}\left(\omega, \theta_{o}^{n}\right)
$$

is made in the statements of Theorem V.1 and Corollary V.1. Here $\Phi_{\varepsilon}\left(\omega, \theta_{o}^{n}\right)$ is to be interpreted as the spectral density of the prediction error residual sequence $\left\{\varepsilon_{t}\left(\theta^{n}\right)\right\}$ defined in (6) evaluated at $\theta^{n}=\theta_{o}^{n}$, the latter being defined in (8).

Proof: The assumption of open-loop data collection only manifests itself in the proofs of Theorem V.1 and Corollary V.1 by allowing the splitting of (35) into two terms in (36), the first of which can have Lemma A.1 applied to it to conclude that $Q_{n} \sim M_{n}\left(\Phi_{u} \Phi_{\nu}\right)+\Delta_{n}$ as $n \rightarrow \infty$ where $\Delta_{n}$ accounts for the second undermodeling-induced term in (36). However, under Gaussian assumptions, and regardless of whether or not the data is collected in open loop, then instead of this strategy Lemma A.2 may be applied directly to establish that $Q_{n} \sim M_{n}\left(\Phi_{u}(\omega) \Phi_{\nu}\left(\omega, \theta_{o}^{n}\right)\right)$ as $n \rightarrow \infty$ (with $\Delta_{n} \sim 0$ ). The rest of the proofs of Theorem V.1 and Corollary V.1 then go through unchanged.

Therefore, in the Gaussian case, the new approximation (53), which contains the pre-existing one (1) as a special FIR case, is shown theoretically to hold for closed-loop data collection. This is the first time a result like (53) has been established for this case of "fixed denominator" modeling, all the preceding work [25], [44], [45], [37] either applying only for open-loop situations, or when considering closed-loop data [22], applies only for the very rare case of the true noise model being $H(q)=1$.

To illustrate the efficacy of Theorem V.2, consider the simulation conditions of Section II altered so that the closedloop scenario of a proportional controller of gain $K=1$ is involved and that furthermore the reference signal $\left\{r_{t}\right\}$ has spectrum $\Phi_{r}(\omega)=0.25 /(1.25-\cos \omega)$ and the measurement noise has spectrum $\Phi_{\nu}(\omega)=(1.36-1.2 \cos \omega) /(1.16-$ $0.8 \cos \omega)$. The sample average and theoretical variabilities given by (1) and (53) for this situation where eight poles in the model structure are not at the origin are shown in Fig. 5. As in Section II, the new approximation (53) shown as the dashed line provides a more informative approximation to the true variability (solid line) than does the pre-existing approximation (1). Note that in using Theorem V.2 to form the (dashed line) approximation in Fig. 5, it was assumed that (since the model structure was considered "rich" and there were no common parameters between dynamics and noise model) there was negligible bias error, and hence that $\Phi_{\varepsilon\left(\theta_{o}^{n}\right)}(\omega) \approx \Phi_{\nu}(\omega)$.

An interesting point is that the simulation producing Fig. 5 specifically violated the Gaussian assumptions that Theorem V.2 was derived under by using uniformly distributed random processes. This robustness of the result to violations of the assumptions it was derived under is considered encouraging from the viewpoint of its practical utility.

\section{BIAS ERROR WITH FIXED DENOMINATOR MODEL STRUCTURE}

Having quantified the variance error involved with using the structure (16), the paper now turns to the question of quantifying the bias error, again by a strategy of considering the equivalent but more tractable orthonormal reparameterization (27). First, a key motivation for a model structure such as (16) is the intuitive belief that for fixed $n$, any undermodeling associated with lack of model structure richness can be decreased by choosing poles $\left\{\xi_{k}\right\}$ in $D_{n}(z)$ close to the true poles $\left\{\gamma_{k}\right\}$ in the underlying true system $G(z)$. The following theorem justifies this intuition by use of the Christoffel-Darboux formula (30).

Theorem VI.1: Suppose $G(z)$ has partial fraction expansion

$$
G(z)=\sum_{i=0}^{m-1} \frac{\alpha_{i}}{z-\gamma_{i}}
$$

where all the poles satisfy $\left|\gamma_{i}\right|<1$. Put $\hat{G}_{n}(z)$ as the best $L_{2}$ approximation to $G(z)$ with respect to the $n$ basis functions $\left\{\mathcal{B}_{0}, \mathcal{B}_{1}, \cdots, \mathcal{B}_{n-1}\right\}$. Then

$$
\left|G\left(e^{j \omega}\right)-\hat{G}_{n}\left(e^{j \omega}\right)\right| \leq \sum_{i=0}^{m-1}\left|\frac{\alpha_{i}}{e^{j \omega}-\gamma_{i}}\right| \prod_{k=0}^{n-1}\left|\frac{\gamma_{i}-\xi_{k}}{1-\overline{\xi_{k}} \gamma_{i}}\right|
$$




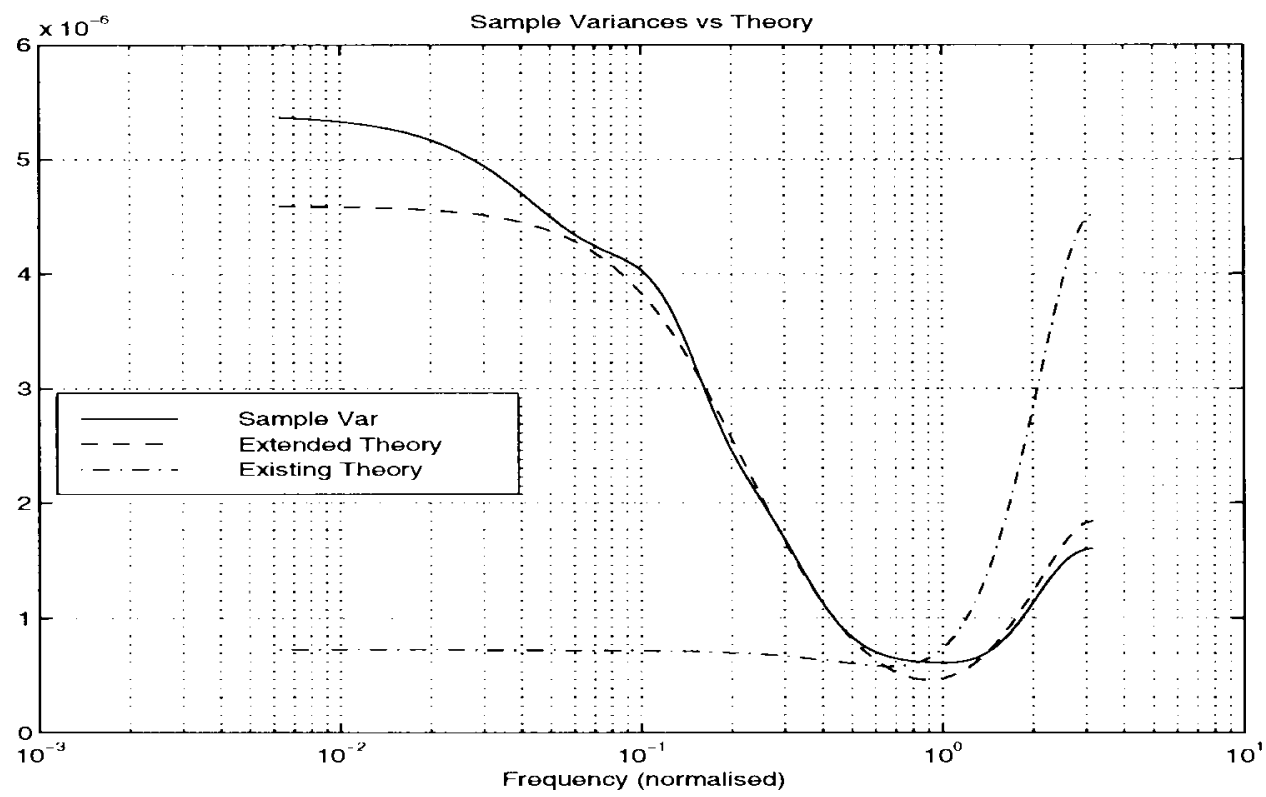

Fig. 5. FIR with eight poles away from origin; closed-loop data collection and a non-Gaussian (uniformly) distributed process.

Proof: Take $\mu \in \mathbf{D}$. Then since $G(1 / z)$ is analytic in $\mathbf{D}$ the use of Cauchy's integral theorem provides

$$
G(1 / \mu)=\frac{1}{2 \pi j} \oint_{\boldsymbol{T}} \frac{G(1 / z)}{z-\mu} d z
$$

Also, by use of the defining property (29) of the reproducing kernel

$$
\hat{G}_{n}(1 / \mu)=\sum_{k=0}^{n-1} \mathcal{B}_{k}(1 / \mu) \frac{1}{2 \pi j} \oint_{T} G(t) \overline{\mathcal{B}_{k}(t)} \frac{d t}{t} .
$$

Using a change of variable $z=t^{-1}$ then leads to

$$
\hat{G}_{n}(1 / \mu)=\frac{1}{2 \pi j} \oint_{\boldsymbol{T}} G(1 / z)\left(\sum_{k=0}^{n-1} \mathcal{B}_{k}(1 / \mu) \overline{\mathcal{B}_{k}(1 / z)}\right) \frac{d z}{z}
$$

so that using the Christoffel-Darboux Formula (30)

$$
\begin{aligned}
G(1 / \mu)-\hat{G}_{n}(1 / \mu) & \\
= & \frac{\mu \varphi_{n}(1 / \mu)}{2 \pi j} \oint_{\boldsymbol{T}} \frac{G(1 / z) \overline{\varphi_{n}(1 / z)}}{z-\mu} \frac{d z}{z} \\
& =\frac{\mu \varphi_{n}(1 / \mu)}{2 \pi j} \oint_{\boldsymbol{T}} \frac{G(z) \overline{\varphi_{n}(z)}}{1-\mu z} d z .
\end{aligned}
$$

Then by using (59) and Cauchy's residue theorem

$$
\begin{aligned}
& G(1 / \mu)-\hat{G}_{n}(1 / \mu) \\
&=\frac{\mu \varphi_{n}(1 / \mu)}{2 \pi j} \sum_{i=0}^{m-1} \oint_{\boldsymbol{T}}\left(\frac{\alpha_{i}}{z-\gamma_{i}}\right)\left(\frac{1}{1-\mu z}\right) \\
& \cdot \prod_{k=0}^{n-1}\left(\frac{z-\xi_{k}}{1-\overline{\xi_{k} z}}\right) d z \\
&= \mu \varphi_{n}(1 / \mu) \sum_{i=0}^{m-1}\left(\frac{\alpha_{i}}{1-\mu \gamma_{i}}\right) \prod_{k=0}^{n-1}\left(\frac{\gamma_{i}-\xi_{k}}{1-\overline{\xi_{k}} \gamma_{i}}\right) .
\end{aligned}
$$

Writing $\mu=r e^{-j \omega}$ with $|r|<1$, letting $r \rightarrow 1$ and using the triangle inequality then gives the result.
Note that this bound is tight in the sense that if perfect knowledge of the poles of $G(z)$ is available (so that $\left.\forall i, \exists k: \xi_{k}=\gamma_{i}\right)$ then the upper bound is zero. The interpretation of the result is that the convergence of the expansion (27) to the underlying system $G(q)$ can be very much faster than that of the special cases of FIR, Laguerre, or two-parameter Kautz expansions if reasonable guesses $\left\{\xi_{k}\right\}$ of the poles of $G(q)$ can be made. This is so since

$$
\frac{z-\xi_{k}}{1-\overline{\xi_{k} z}}
$$

is analytic on $\mathrm{D}$ and of modulus 1 on the boundary $\boldsymbol{T}$ of D. Therefore, by the maximum modulus theorem, the factor (60) with $z=\gamma_{i}$ that appears $n$ times in the bound (58) is of modulus less than one, and hence the bound (58) decreases geometrically with model order $n$; the geometric rate will depend on the error term $\left|\gamma_{i}-\xi_{i}\right|$ in the guess $\xi_{i}$ in the true pole position $\gamma_{i}$.

A bound similar to that of Theorem VI.1 is given, using completely different methods related to the new theory of "Hambo" transforms in [15]. However, it is less explicit since it contains a constant scaling factor for which the only information available is that it is finite.

The significance of Theorem VI.1 in the context of quantifying the undermodeling-induced error in an estimated frequency response is realized by noting that

$$
\theta_{o}^{n}=\underset{\theta^{n} \in \boldsymbol{R}^{n}}{\arg \min }\left\{\int_{-\pi}^{\pi}\left|G\left(e^{j \omega}\right)-G\left(e^{j \omega}, \theta^{n}\right)\right|^{2} \Phi_{u}(\omega) d \omega\right\}
$$

so that for $\left\{u_{t}\right\}$ white $\left(\Phi_{u}(\omega)=\right.$ constant), the expression (58) is a quantification of the asymptotic undermodelinginduced estimation error.

Although (58) appears to be the most explicit statement that can be made about undermodeling-induced error, it is 
possible to draw further conclusions that apply also when $\Phi_{u}(\omega)$ is not white. For example, it is shown in [31] that the frequency response magnitude is on average (over frequency) underestimated in the sense that

$$
\int_{-\pi}^{\pi}\left|G\left(e^{j \omega}, \theta_{o}^{n}\right)\right|^{2} \Phi_{u}(\omega) d \omega \leq \int_{-\pi}^{\pi}\left|G\left(e^{j \omega}\right)\right|^{2} \Phi_{u}(\omega) d \omega .
$$

Equation (61) may also be used to bound the bias error in estimating $G\left(e^{j \omega}\right)$ by noting that since, under the condition $\Sigma\left(1-\left|\xi_{k}\right|\right)=\infty$ the $\left\{\mathcal{B}_{k}\right\}$ are complete in $H_{2}(T)$, then it may be expanded as

$$
G\left(e^{j \omega}\right)=\sum_{\ell=0}^{n-1} \theta_{\ell}^{n} \mathcal{B}_{\ell}\left(e^{j \omega}\right)+\sum_{\ell=n}^{\infty} \kappa_{\ell} \mathcal{B}_{\ell}\left(e^{j \omega}\right)
$$

Using this notation, the following bias error bound can be obtained.

Lemma VI.1: Suppose that $G(z) \in H_{2}(\boldsymbol{T})$ so that the coefficients $\kappa_{\ell}$ in (62) are well defined. Then

$$
\begin{aligned}
\mid G\left(e^{j \omega}\right) & -G\left(e^{j \omega}, \theta_{o}^{n}\right) \mid \\
\leq & \left(n \frac{\max _{\omega} \Phi_{u}(\omega)}{\min _{\omega} \Phi_{u}(\omega)}+1\right) \times \max _{0 \leq k<n}\left|\mathcal{B}_{k}(\omega)\right| \\
& \cdot \sum_{\ell=n}^{\infty}\left|\kappa_{\ell}\right| \cdot \quad \text { w.p.1. }
\end{aligned}
$$

Proof: The method of proof is identical to that employed by Wahlberg in [44]. Expression (61) characterizes $\theta_{o}^{n}$ as the solution to

$$
R_{n} \theta_{o}^{n}=\mathbf{E}\left\{\phi_{k} y_{k}\right\}
$$

Using this and defining the sequence $\left\{z_{k}\right\}$ as

$$
z_{k}=\sum_{\ell=n}^{\infty} \kappa_{\ell} \mathcal{B}_{\ell}(q) u_{k}
$$

gives

$$
\left\|\theta^{n}-\theta_{o}^{n}\right\|_{2} \leq\left\|R_{n}^{-1}\right\|_{2}\left\|\mathbf{E}\left\{\phi_{k} z_{k}\right\}\right\|_{2} .
$$

But by Parseval's theorem

$$
\left[\mathbf{E}\left\{\phi_{k} z_{k}\right\}\right]_{m}=\frac{1}{2 \pi} \sum_{\ell=n}^{\infty} \kappa_{\ell} \int_{-\pi}^{\pi} \mathcal{B}_{m}\left(e^{j \omega}\right) \overline{\mathcal{B}_{\ell}\left(e^{j \omega}\right)} \Phi_{u}(\omega) d \omega .
$$

Now, using the triangle inequality, the Cauchy-Schwarz inequality, and using (33) to bound $\left\|R_{n}^{-1}\right\|$ provides

$$
\left\|\theta^{n}-\theta_{o}^{n}\right\|_{1} \leq n \frac{\max _{\omega} \Phi_{u}(\omega)}{\min _{\omega} \Phi_{u}(\omega)} \sum_{\ell=n}^{\infty}\left|\kappa_{\ell}\right|
$$

But from (62)

$$
\begin{aligned}
& \left|G\left(e^{j \omega}\right)-G\left(e^{j \omega}, \theta_{o}^{n}\right)\right| \\
& \quad \leq \max _{0 \leq k<n}\left|\mathcal{B}_{k}\left(e^{j \omega}\right)\right|\left(\left\|\theta^{n}-\theta_{o}^{n}\right\|_{1}+\sum_{\ell=n}^{\infty}\left|\kappa_{\ell}\right|\right) .
\end{aligned}
$$

As mentioned for the particular case of Laguerre models studied in [44], this indicates that as $n \rightarrow \infty$ then $\theta_{o}^{n}$ will converge to the expansion coefficients given in (62) and hence the frequency response estimate will converge to the true one provided the expansion coefficients in (62) decrease sufficiently quickly so that

$$
\lim _{n \rightarrow \infty} n \sum_{\ell=n}^{\infty}\left|\kappa_{\ell}\right|=0
$$

This is satisfied, for example, if $G(z)$ is stable and finite dimensional. In this case a simple argument using Cauchy's residue theorem shows that there exists a $|\eta|<1$ and $K<\infty$ such that $\left|\kappa_{\ell}\right|<K \eta^{\ell}$.

\section{ARX-TyPE MODEL STRUCTURES}

Although the fixed denominator model structure (16) and its generalization (54), (55) have many practical advantages such as relevance to "identification for control" ideas [1], [40], [27], [33] and the requirement of only simple and robust numerical procedures for the calculation of $\hat{\theta}_{N}^{n}$ [44], [37], they suffer from the drawback of relying on prior knowledge of pole location.

A common strategy for avoiding this drawback is to estimate the pole locations of $G(q)$ while still involving a predictor that is linear in $\theta^{n}$ so that the advantage of simple numerical requirements for finding $\hat{\theta}_{N}^{n}$ is retained. This is done by employing the model structure

$$
G\left(q, \theta^{n}\right)=\frac{B\left(q, \theta^{n}\right)}{A\left(q, \theta^{n}\right)}, \quad H\left(q, \theta^{n}\right)=\frac{D_{n}(q)}{A\left(q, \theta^{n}\right)}
$$

where

$$
\begin{aligned}
& A(q)=a_{0}+a_{1} q+a_{2} q^{2}+\cdots+a_{n-1} q^{n-1}+q^{n} \\
& B(q)=b_{0}+b_{1} q+b_{2} q^{2}+\cdots+b_{n-1} q^{n-1}
\end{aligned}
$$

with

$$
\theta^{n}=\left[a_{0}, b_{0}, a_{1}, b_{1}, \cdots, a_{n-1}, b_{n-1}\right]^{T}
$$

being the vector of parameters to be estimated and $D_{n}(q)$ is as previously defined in (17).

Here the dynamics and noise model share parameters in $\theta^{n}$. As is well known, this can lead to bias if the model structure is not rich enough [46], [20]. The motivation for including the $D_{n}(q)$ term in the noise model is to avoid this bias by allowing $H\left(q, \theta_{o}^{n}\right) \approx H(q)$ for some $\theta_{o}^{n}$ while simultaneously, through zeros of $B\left(q, \theta_{o}^{n}\right)$ cancelling parts of $A\left(q, \theta_{o}^{n}\right)$ that pertain only to $H\left(q, \theta_{o}^{n}\right)$, achieving sufficient flexibility for $G\left(q, \theta_{o}^{n}\right)=G(q)$.

Most commonly the model structure (63) appears with the choice $\xi_{k}=0$ in which case it is known as the "equation error" or sometimes "ARX" model structure and for which the analysis of Ljung [22] provides, using the definition (12), the well-known result (which holds, when at least asymptotically in $n$, the true system is in the model set)

$$
\operatorname{Cov}\left\{\Pi\left(e^{j \omega}, \hat{\theta}_{N}^{n}\right)\right\} \approx \frac{n}{N} \sigma^{2}\left|H\left(e^{j \omega}, \theta_{o}^{n}\right)\right|^{2} \Phi_{\zeta}^{-1}\left(e^{j \omega}, \theta_{o}^{n}\right) .
$$

Here

$$
\Phi_{\zeta}\left(\omega, \theta^{n}\right) \triangleq\left[\begin{array}{cc}
\Phi_{u}(\omega) & \Phi_{u \varepsilon\left(\theta^{n}\right)}^{*}(\omega) \\
\Phi_{u \varepsilon\left(\theta^{n}\right)}(\omega) & \Phi_{\varepsilon\left(\theta^{n}\right)}(\omega)
\end{array}\right]
$$




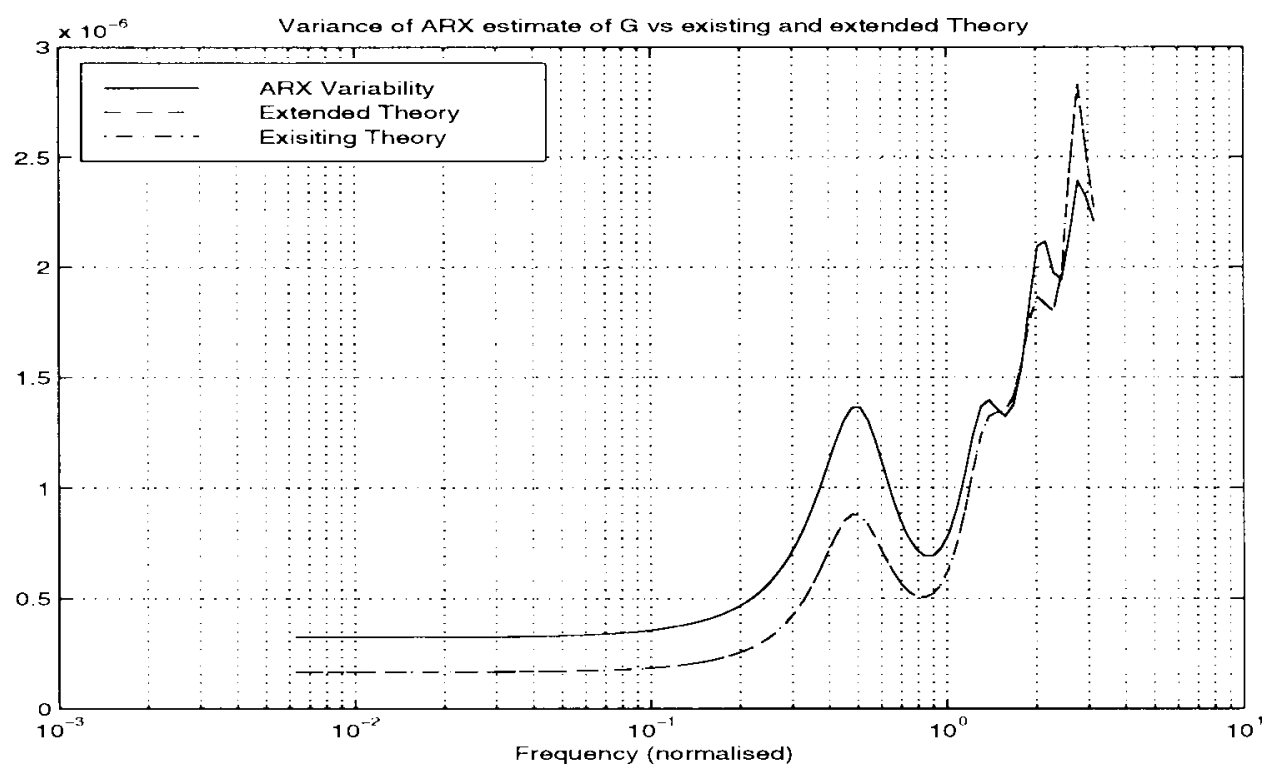

Fig. 6. Conventional ARX estimation with all noise model zeros at the origin. This is a comparison of the Monte Carlo estimate of sample variability (solid line) with (dash-dot line) the approximate expression (64). Note that this last line obscures a dashed line which is the new approximation (79) because for the case of all poles at the origin the pre-existing approximation (64) and the new one (79) are identical.

is the spectral density of the two-dimensional vector signal $\zeta_{t}\left(\theta^{n}\right)$ defined as

$$
\zeta_{t}\left(\theta^{n}\right) \triangleq\left[\begin{array}{c}
u_{t} \\
\varepsilon_{t}\left(\theta^{n}\right)
\end{array}\right]
$$

The importance of the latter, as will become clear in a moment, is to allow [by use of (5)] the prediction error gradient $\psi_{t}\left(\theta^{n}\right)$ to be expressed as

$$
\begin{aligned}
\psi_{t}\left(\theta^{n}\right) & =\frac{d \hat{y}_{t}\left(\theta^{n}\right)}{d \theta^{n}}=H^{-1}\left(q, \theta^{n}\right) \Pi^{\prime}\left(q, \theta^{n}\right) \zeta_{t}\left(\theta^{n}\right) \\
\Pi^{\prime}\left(q, \theta^{n}\right) & \triangleq\left[\frac{d G\left(q, \theta^{n}\right)}{d \theta^{n}}, \frac{d H\left(q, \theta^{n}\right)}{d \theta^{n}}\right] .
\end{aligned}
$$

Since this strategy of avoiding bias can lead to overparameterization of $G\left(q, \theta^{n}\right)$, the degree of which depends on how many extra terms in $A\left(q, \theta^{n}\right)$ are required for modeling $H(q)$, there is great importance in choosing the zeros $\left\{\xi_{k}\right\}$ not, in fact, all at the origin but as close as possible to where the true zeroes of $H(q)$ are believed to lie. For example, if $H(q)$ has a zero near the unit circle, an AR expansion $q^{n} / A\left(q, \theta^{n}\right)$ accounting for this zero will need to be of quite high order $n$ before it is accurate [39].

A main purpose of this section is to highlight that unfortunately (and in resonance with the fixed denominator case) if according to this motivation the $\left\{\xi_{k}\right\}$ are not all chosen at the origin, then the approximation (64) can be quite inaccurate, even for large model order and data length.

This perhaps unexpected phenomenon can be illustrated in a fashion similar to that of Section II by considering the least squares method (7) and an $n=8$ th-order ARX-like structure (63) for the estimation of the "Åström system"

$$
G(q)=\frac{q+0.5}{q^{2}-1.5 q+0.7}, \quad H(q)=1
$$

on the basis of $N=10000$ observed open-loop input-output measurements, the former being white Gaussian noise with spectral density $\Phi_{u}(\omega)=0.25 /(1.25-\cos \omega)$ and the latter being corrupted by white Gaussian noise of variance $\sigma^{2}=$ 0.001 . Suppose also that all fixed noise model zeroes $\left\{\xi_{k}\right\}$ in $D_{n}(q)$ are chosen at the origin, so that a true ARX structure is employed. Note that in this example, and all the rest following in this section, the bias error in the estimation process is negligible, and hence the variance error will represent the total estimation error. In any event, since both $N=10000$ and $n=8$ can reasonably be considered large [22], then the approximation (64) for the variance error could be expected to be accurate, and indeed it appears to be so when shown as the dash-dot line in Fig. 6, with the sample average (over 500 Monte Carlo simulations) estimate of the true variability being shown as the solid line.

However, if three noise model zeros are moved away from the origin to be at $\xi_{k}=\{0.8,0.8,0.8\}$, then the ensuing comparison of the theoretical (dash-dot line) approximation (64) and the Monte Carlo estimate (solid line) of true variability shown in Fig. 7 shows much less agreement. Continuing, by choosing six noise model zeros away from the origin at $\left\{\xi_{k}\right\}=$ $\{0.8,0.8,0.8,0.7,0.7,0.7\}$, the results of this choice shown in Fig. 8 indicate that now the approximation between (dash-dot line) the theoretical approximation (64) and (solid line) the estimated true variability is so poor as to be considered very uninformative.

In contrast, the dashed line in Figs. 6-8 (which, in Fig. 6) is equal to and hence obscured by the dash-dot line) remains a good approximation regardless of the fixed zero position. This line is in fact the new approximation to be derived in this section, and again in resonance with the preceding fixed denominator analysis, it involves replacing the $n$ term in (64) with a term $K_{n}(\omega, \omega)$. Since the latter in fact equals $n$ for 


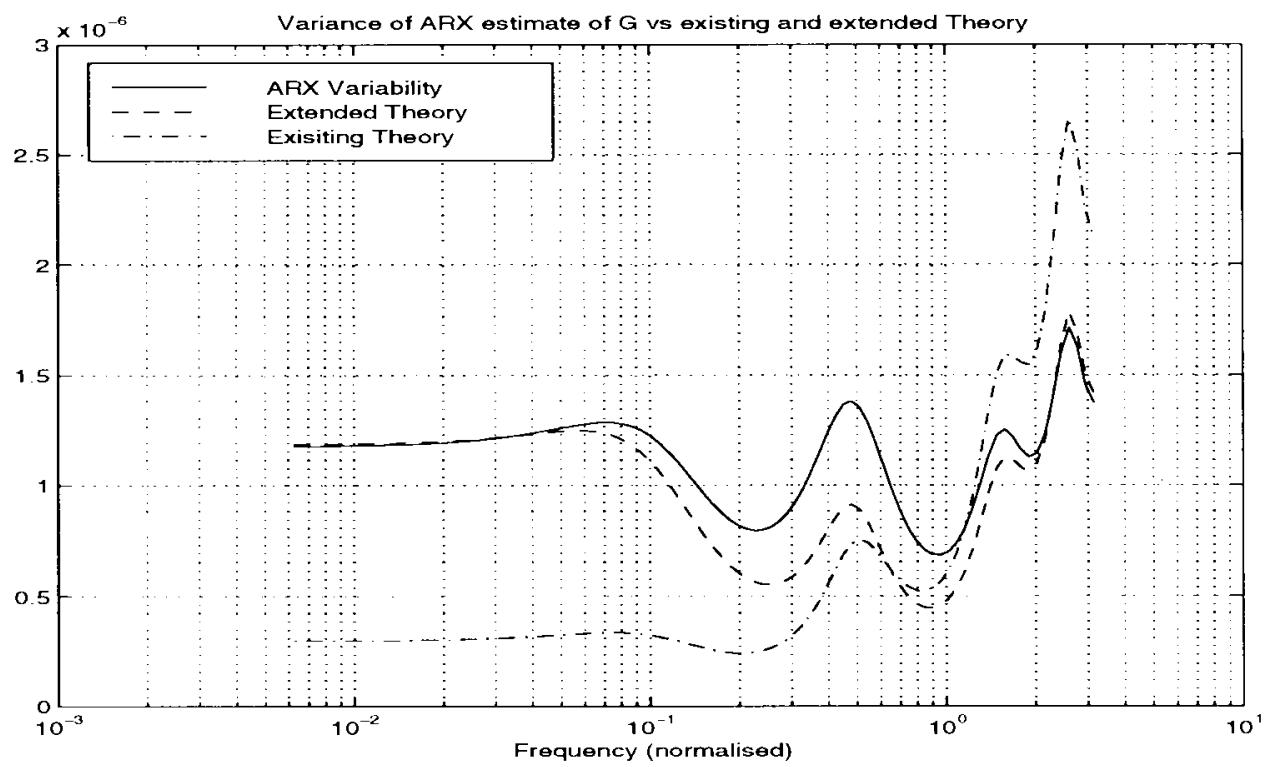

Fig. 7. ARX-type structure with three noise model zeros not at the origin; comparison of Monte Carlo estimate of sample variability (solid line) with (dash-dot line) the approximate expression (64) and (dashed line) the new approximation (79).

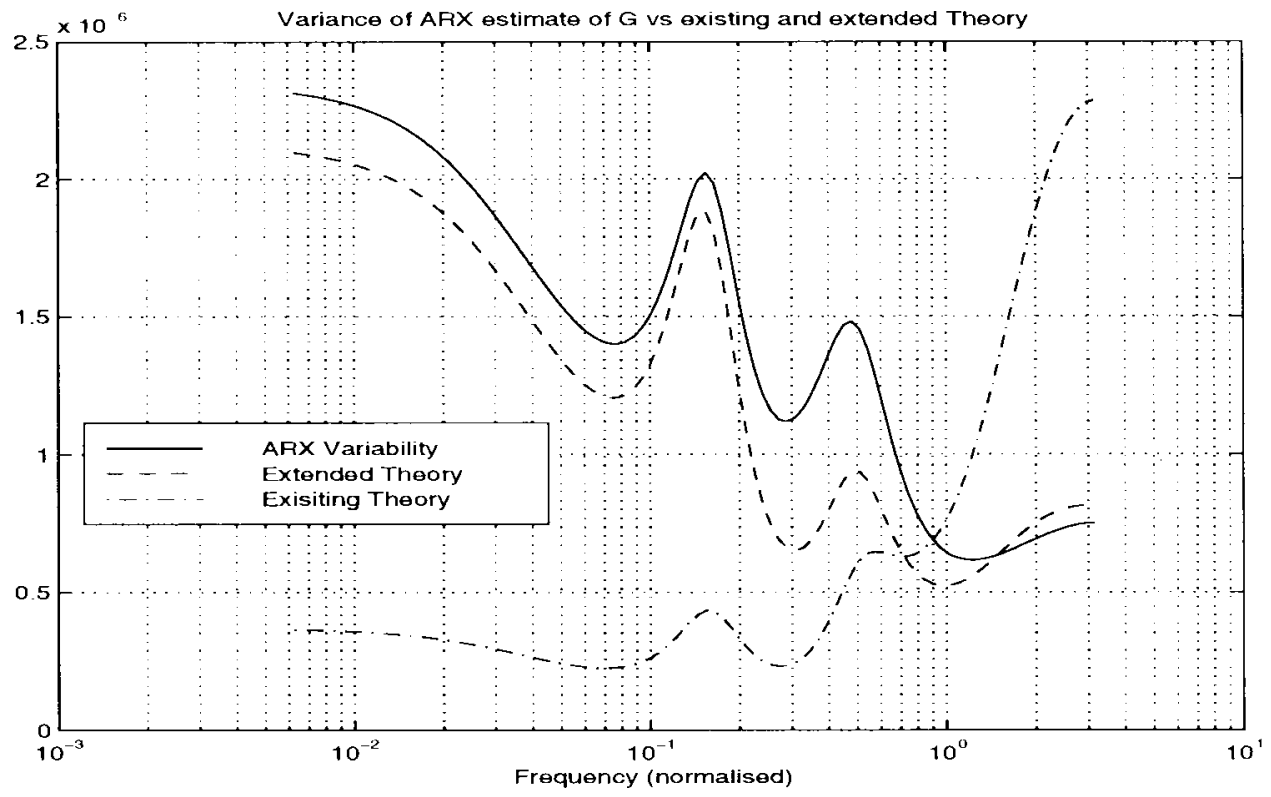

Fig. 8. ARX-type structure with six noise model zeros not at the origin.

all the zeros at the origin, the original approximation (64) is encompassed as a special case.

Also in accordance with the previous analysis, the improved approximation shown in Figs. 6-8 is obtained by reparameterizing the model structure into an equivalent orthonormal form which is tractable to analysis using the new generalized Fourier and Toeplitz results of [35] presented in Section V.

It is, of course, first necessary to establish the theoretical genesis of the inaccuracy phenomenon illustrated in Figs. 6-8, and for this purpose it is necessary to give a brief synopsis of the methods used in [22] to derive (64).

The conditions assumed there which are salient to the model structure are that it satisfy what is called a "shift" property formulated as the requirement that with $\Gamma_{n}(q)$ defined as in (18), an $s \times s$ matrix of transfer functions $Z\left(q, \theta^{n}\right)$ exists such that

$$
\Pi^{\prime}\left(q, \theta^{n}\right)=\left[\Gamma_{n}(q) \otimes I_{s}\right] Z\left(q, \theta^{n}\right)
$$

where the dimension $s$ will be made clear in a moment by example and $\otimes$ denotes the Kronecker tensor product of matrices defined for an $m \times n$ matrix $A$ and an $\ell \times p$ matrix $B$ to provide the $n \ell \times m p$ matrix $A \otimes B$ as

$$
A \otimes B \triangleq\left(\begin{array}{cccc}
a_{11} B & a_{12} B & \cdots & a_{1 n} B \\
a_{21} B & a_{22} B & \cdots & a_{2 n} B \\
\vdots & & & \vdots \\
a_{m 1} B & a_{m 2} B & \cdots & a_{m n} B
\end{array}\right) .
$$


Furthermore, it is argued in [22] that assuming that $\theta_{o}^{n}$ is such that $G\left(q, \theta_{o}^{n}\right)=G(q), H\left(q, \theta_{o}^{n}\right)=H(q)$ (that is, it is assumed that the true system is in the model set) then with the Toeplitz matrix definition (18), (20) extended to block-Toeplitz matrices via

$$
T_{n}(F) \triangleq \frac{1}{2 \pi} \int_{-\pi}^{\pi}\left[\Gamma_{n}(\omega) \otimes I_{s}\right] F(\omega)\left[\Gamma_{n}^{*}(\omega) \otimes I_{s}\right] d \omega
$$

to handle $s \times s$ multivariable spectral densities $F(\omega)$, then using known results on the asymptotic nature of block-Toeplitz matrices [49], [14]

$$
\begin{aligned}
P_{n} \sim \sigma^{2} T_{n}\left(\left|H\left(e^{j \omega}, \theta_{0}^{n}\right)\right|^{2}[\right. & Z\left(e^{j \omega}, \theta_{o}^{n}\right) \Phi_{\zeta}\left(\omega, \theta_{o}^{n}\right) \\
& \left.\left.Z^{*}\left(e^{j \omega}, \theta_{o}^{n}\right)\right]^{-1}\right) \\
& \text { as } n \rightarrow \infty
\end{aligned}
$$

so that recognizing implicit Cesáro means and employing classical results from Fourier analysis via (14), it is argued in [22] that for large $n$ the following approximations are valid (some abuse of notation is made in the interests of readability):

$$
\begin{aligned}
\frac{\Lambda_{n}(\omega)}{n}= & n^{-1}\left[\Pi \Pi^{\prime}\left(e^{j \omega}, \theta_{o}^{n}\right)\right]^{*} P_{n} \Pi^{\prime}\left(e^{j \omega}, \theta_{o}^{n}\right) \\
\approx & n^{-1} Z_{o}^{*}(\omega)\left[\Gamma_{n}^{*}(\omega) \otimes I_{s}\right] \\
& \times \sigma^{2} T_{n}\left(\left|H_{0}(\omega)\right|^{2}\left[Z_{o}(\omega) \Phi_{\zeta}\left(\omega, \theta_{o}^{n}\right) Z_{o}^{*}(\omega)\right]^{-1}\right) \\
& \times\left[\Gamma_{n}(\omega) \otimes I_{s}\right] Z_{o}(\omega) \\
\approx & \sigma^{2}\left|H_{o}(\omega)\right|^{2} Z_{o}^{*}(\omega)\left[Z_{o}(\omega) \Phi_{\zeta}\left(\omega, \theta_{o}^{n}\right) Z_{o}^{*}(\omega)\right]^{-1} \\
& \cdot Z_{o}(\omega) \\
= & \sigma^{2}\left|H\left(e^{j \omega}, \theta_{o}^{n}\right)\right|^{2} \Phi_{\zeta}^{-1}\left(\omega, \theta_{o}^{n}\right)
\end{aligned}
$$

where in progressing to the last line, invertibility of $Z_{o}(\omega)$ has been assumed.

However, for the model structure (63), it is easily shown [22] that with the elements in the vector $\theta^{n}$ grouped into $n$ blocks each of dimension $s=2$ and each of the form $\theta_{k}^{n}=\left[a_{k}, b_{k}\right]^{T}$, then the corresponding $Z\left(q, \theta^{n}\right)$ of (66) is given as

$$
Z\left(q, \theta^{n}\right)=\frac{1}{D_{n}(q)}\left[\begin{array}{cc}
-G\left(q, \theta^{n}\right) H\left(q, \theta^{n}\right) & -H^{2}\left(q, \theta^{n}\right) \\
H\left(q, \theta^{n}\right) & 0
\end{array}\right] .
$$

Therefore, when evaluated at $\theta_{o}^{n}$ it is better labeled as $Z_{o}(\omega, n)$ since if all the $\xi_{k}$ are chosen away from the origin then via the $D_{n}^{-1}(q)$ term, the magnitude of the transfer function components of $Z\left(e^{j \omega}, \theta_{o}^{n}\right)$ all depend on $n$. In this case the Fourier convergence argument allowing the progression from (69), (70) is problematic since the matrix-valued function $\left[Z_{o}(\omega, n) \Phi_{\zeta}\left(\omega, \theta_{o}^{n}\right) Z_{o}^{*}(\omega, n)\right]^{-1}$ whose $n$ th-order Fourier reconstruction is implicit in (69), cannot be guaranteed to converge as $n$ increases since the function possibly (depending on how many zeros of $D_{n}(q)$ are not at the origin) becomes less smooth with increasing $n$.

In the strict ARX case originally analyzed in [22] there is (as illustrated in Fig. 6) no problem since in this instance, with all the zeros at the origin, $\left.\left|D_{n}\left(e^{j \omega}\right)\right|=\mid e^{j \omega n}\right) \mid=1$ so that by the assumption of the true system being in the model class, then the function $Z_{o}(\omega) \Phi_{\zeta}\left(\omega, \theta_{o}^{n}\right) Z_{o}^{*}(\omega)$ being implicitly Fourier reconstructed in (69) is invariant to increasing $n$, so that indeed it is valid to conclude increasing accuracy with increasing $n$ and hence pass from (69), (70).

Therefore, in order to provide the improved approximation shown in Figs. 6-8 for the case of many zeros of $D_{n}(q)$ not at the origin, the challenge is to mimic this invariant to $n$ behavior of the underlying Fourier reconstructed function.

As in Section V, this is achieved by using the observation that, asymptotically in $n$, the frequency response properties of estimates are invariant to (injective) changes in model structure, so that instead of the ARX-like structure (63) the equivalent orthonormally parameterized model structure

$$
\begin{aligned}
G\left(q, \theta^{n}\right) & =\frac{B\left(q, \theta^{n}\right)}{A\left(q, \theta^{n}\right)} \\
& =\left[\sum_{k=0}^{n-1} b_{k} \mathcal{B}_{k}(q)\right]\left[1-\sum_{k=0}^{n-1} a_{k} \mathcal{B}_{k}(q)\right]^{-1} \\
H\left(q, \theta^{n}\right) & =\frac{1}{A\left(q, \theta^{n}\right)}=\left[1-\sum_{k=0}^{n-1} a_{k} \mathcal{B}_{k}(q)\right]
\end{aligned}
$$

is considered, whether or not the actual implementation employs this or the more natural form (63).

The advantage of the orthonormally parameterized form (71), (72) is that with the generalized definition (32) for $\Gamma_{n}(q)$, it satisfies a generalized shift property (again with $s=2$ )

$$
\Pi^{\prime}\left(q, \theta^{n}\right)=\left[\Gamma_{n}(q) \otimes I_{s}\right] Z\left(q, \theta^{n}\right)
$$

where now

$$
Z\left(q, \theta_{o}^{n}\right)=\left[\begin{array}{cc}
-G\left(q, \theta_{o}^{n}\right) H\left(q, \theta_{o}^{n}\right) & -H^{2}\left(q, \theta_{o}^{n}\right) \\
H\left(q, \theta_{o}^{n}\right) & 0
\end{array}\right]
$$

is (by the assumption of true system lying in the model class) independent of $n$ for any choice of $\left\{\xi_{k}\right\}$.

To make this more precise, it is necessary to expand (31) to the definition of a generalized block-Toeplitz matrix depending on an $s \times s$ dimensional positive definite matrixvalued function $F(\omega)$ as

$$
M_{n}(F) \triangleq \frac{1}{2 \pi} \int_{-\pi}^{\pi}\left[\Gamma_{n}(\omega) \otimes I_{s}\right] F(\omega)\left[\Gamma_{n}^{*}(\omega) \otimes I_{s}\right] d \omega
$$

where $\Gamma_{n}(q)$ is the generalized form (32). Also, using the assumption that (at least asymptotically as $n \rightarrow \infty$ ) the true system is in the model set [22], then $\varepsilon_{t}\left(\theta_{o}^{n}\right)=e_{t}$ so that using (65), (10), (11) and Parseval's theorem

$$
\begin{aligned}
& R_{n}=M_{n}\left(\frac{Z\left(e^{j \omega}, \theta_{o}^{n}\right) \Phi_{\zeta}\left(\omega, \theta_{o}^{n}\right) Z^{*}\left(e^{j \omega}, \theta_{o}^{n}\right)}{\left|H\left(e^{j \omega}, \theta_{o}^{n}\right)\right|^{2}}\right) \\
& Q_{n}=\sigma^{2} R_{n}
\end{aligned}
$$

and therefore

$$
\begin{aligned}
P_{n} & =\sigma^{2} R_{n}^{-1} \\
& =\sigma^{2} M_{n}^{-1}\left(\frac{Z\left(e^{j \omega}, \theta_{o}^{n}\right) \Phi_{\zeta}\left(\omega, \theta_{o}^{n}\right) Z^{*}\left(e^{j \omega}, \theta_{o}^{n}\right)}{\left|H\left(e^{j \omega}, \theta_{o}^{n}\right)\right|^{2}}\right) .
\end{aligned}
$$

However, by Lemma A.5 the same equivalence (45) shown in [35] to hold for generalized Toeplitz matrices can also 
be shown to hold for generalized block Toeplitz matrices. In particular, using the definition (74)

$$
\begin{aligned}
M_{n}^{-1} & \left(\frac{Z\left(e^{j \omega}, \theta_{o}^{n}\right) \Phi_{\zeta}\left(\omega, \theta_{o}^{n}\right) Z^{*}\left(e^{j \omega}, \theta_{o}^{n}\right)}{\left|H\left(e^{j \omega}, \theta_{o}^{n}\right)\right|^{2}}\right) \\
\sim & M_{n}\left(| H ( e ^ { j \omega } , \theta _ { o } ^ { n } ) | ^ { 2 } \left[Z\left(e^{j \omega}, \theta_{o}^{n}\right) \Phi_{\zeta}\left(\omega, \theta_{o}^{n}\right)\right.\right. \\
& \left.\left.\cdot Z^{*}\left(e^{j \omega}, \theta_{o}^{n}\right)\right]^{-1}\right)
\end{aligned}
$$

as $n \rightarrow \infty$ where here the definition of the equivalence is extended from that of (39), (40) to being that (75) implies that

$$
\begin{aligned}
\lim _{n \rightarrow \infty} & K_{n}^{-1}(\omega, \omega)\left[\Gamma_{n}^{*}(\omega) \otimes I_{s}\right]\left[M_{n}\left(F^{-1}\right)-M_{n}^{-1}(F)\right] \\
\times & {\left[M_{n}\left(F^{-1}\right)-M_{n}^{-1}(F)\right]^{T}\left[\Gamma_{n}(\omega) \otimes I_{s}\right]=0 }
\end{aligned}
$$

component-wise and for all $\omega \in[-\pi, \pi]$. Therefore, again using the reasoning in (41), then from (14) use of the orthonormal reparameterization idea means that instead of (69) the following expression is considered:

$$
\begin{aligned}
\lim _{n \rightarrow \infty} & \frac{\Lambda_{n}(\omega)}{K_{n}(\omega, \omega)} \\
= & \lim _{n \rightarrow \infty} \frac{1}{K_{n}(\omega, \omega)} Z_{o}^{*}(\omega)\left[\Gamma_{n}^{*}(\omega) \otimes I_{s}\right] \\
& \times M_{n}\left(\left|H_{o}(\omega)\right|^{2}\left[Z_{o}(\omega) \Phi_{\zeta}\left(\omega, \theta_{o}^{n}\right) Z_{o}^{*}(\omega)\right]^{-1}\right) \\
& \times\left[\Gamma_{n}(\omega) \otimes I_{s}\right] Z_{o}(\omega)+\frac{o\left(\left\|\hat{\theta}_{N}^{n}-\theta_{o}^{n}\right\|^{2}\right)}{K_{n}(\omega, \omega)} .
\end{aligned}
$$

Asymptotic in $n$ analysis of this expression then provides what are the last main technical results of the paper.

Theorem VII.1: With $\hat{\theta}_{N}^{n}$ calculated via (7) using the model structures (63), (71), (72) or any other equivalent structure with the same fixed noise model zeroes $\left\{\xi_{k}\right\}$ all chosen to lie within the unit disk $\mathrm{D}$, then in the limit as $N \rightarrow \infty$

$$
\sqrt{N}\left(\begin{array}{c}
G\left(e^{j \omega}, \hat{\theta}_{N}^{n}\right)-G\left(e^{j \omega}, \theta_{o}^{n}\right) \\
H\left(e^{j \omega}, \hat{\theta}_{N}^{n}\right)-H\left(e^{j \omega}, \theta_{o}^{n}\right)
\end{array}\right) \stackrel{\mathcal{D}}{\rightarrow} \mathcal{N}\left(0, \Lambda_{n}(\omega)\right)
$$

where with $K_{n}(\omega, \omega) \equiv K_{n}\left(e^{j \omega}, e^{j \omega}\right)$ given by (28) and under the assumption that eventually, for large enough $n$, the true system is in the model set, then

$$
\lim _{n \rightarrow \infty} \frac{\Lambda_{n}(\omega)}{K_{n}(\omega, \omega)}=\Phi_{\nu}(\omega)\left[\begin{array}{cc}
\Phi_{u}(\omega) & \Phi_{u e}(\omega) \\
\Phi_{u e}^{*}(\omega) & \sigma^{2}
\end{array}\right]^{-1}
$$

Proof: It has already been established in (13) that with the definition of the composite transfer matrix (12)

$$
\sqrt{N}\left[\Pi\left(e^{j \omega}, \hat{\theta}_{N}^{n}\right)-\Pi\left(e^{j \omega}, \hat{\theta}_{o}^{n}\right)\right] \stackrel{\mathcal{D}}{\rightarrow} \mathcal{N}\left(0, \Lambda_{n}(\omega)\right) \quad \text { as } N \rightarrow \infty
$$

where (76) gives an asymptotic in $n$ expression for $\Lambda_{n}(\omega) / K_{n}(\omega, \omega)$. Because of the Kronecker product terms in (76), the result (50) is not applicable. However, Lemma A.3 shows that for $F(\omega)$ an arbitrary regular multivariable spectral density, then (50) may be extended to

$$
\lim _{n \rightarrow \infty} \frac{1}{K_{n}(\omega, \omega)}\left[\Gamma_{n}^{*}(\omega) \otimes I_{s}\right] M_{n}(F)\left[\Gamma_{n}(\omega) \otimes I_{s}\right]=F(\omega)
$$

component-wise in $F(\omega)$ and where $M_{n}(F)$ is defined by (67). Applying this result to (76) with $F(\omega)=$
$\left|H_{o}(\omega)\right|^{2}\left[Z_{o}(\omega) \Phi_{\zeta}\left(\omega, \theta_{o}^{n}\right) Z_{o}^{*}(\omega)\right]^{-1}$ then gives (since $Z\left(e^{j \omega}, \theta_{o}^{n}\right)$ is invertible)

$$
\begin{aligned}
\lim _{n \rightarrow \infty} & \frac{\Lambda_{n}(\omega)}{K_{n}(\omega, \omega)} \\
\quad= & \left|H_{o}(\omega)\right|^{2} Z_{o}^{*}(\omega)\left[Z_{o}(\omega) \Phi_{\zeta}\left(\omega, \theta_{o}^{n}\right) Z_{o}^{*}(\omega)\right]^{-1} Z_{o}(\omega) \\
\quad & =\sigma^{2}\left|H\left(e^{j \omega}, \theta_{o}^{n}\right)\right|^{2} \Phi_{\zeta}^{-1}\left(\omega, \theta_{o}^{n}\right) .
\end{aligned}
$$

Corollary VII.1: Under the same conditions as the previous theorem, but with a strengthened requirement that $\mathbf{E}\left\{e_{k}^{8}\right\}<\infty$, then

$$
\begin{array}{r}
\lim _{N, n \rightarrow \infty} \frac{N}{K_{n}(\omega, \omega)} \operatorname{Cov}\left\{\Pi\left(e^{j \omega}, \hat{\theta}_{N}^{n}\right)\right\} \\
=\Phi_{\nu}(\omega)\left[\begin{array}{cc}
\Phi_{u}(\omega) & \Phi_{u e}(\omega) \\
\Phi_{u e}^{*}(\omega) & \sigma^{2}
\end{array}\right]^{-1} .
\end{array}
$$

Proof: The proof follows from the previous theorem using the methods in [20, Appendix 9B].

The essential points of Theorem VII.1 and Corollary VII.1 that discriminates them from previous corresponding results [22] is that the asymptotic expression (76a) implicitly involves generalized Fourier reconstruction of a matrix-valued function $\left|H_{o}(\omega)\right|^{2}\left[Z_{o}(\omega) \Phi_{\zeta}\left(\omega, \theta_{o}^{n}\right) Z_{o}^{*}(\omega)\right]^{-1}$ which is invariant to $n$, regardless of whether fixed noise model zeros are chosen at the origin or not. In this case, and unlike the analysis leading to (64), it can be expected that the approximation between the left-hand side of (76a) and its asymptotic value on the righthand side of (76a) will monotonically improve with increasing $n$, again regardless of the location of the fixed noise model zeros.

As a consequence of this, the suggestion of this paper is that an improved approximation for the variability of ARX-type model structure estimates is obtained from (52) by assuming that for finite $N$ and $n$ convergence has approximately converged in (76a) so that

$$
\operatorname{Cov}\left\{\Pi\left(e^{j \omega}, \hat{\theta}_{N}^{n}\right)\right\} \approx \frac{1}{N} \Phi_{\nu}(\omega) \Phi_{\zeta}^{-1}\left(\omega, \theta_{o}^{n}\right) K_{n}(\omega, \omega) .
$$

In particular, if the data is collected in open-loop $\left(\Phi_{u e}(\omega)=0\right)$ then

$$
\operatorname{Cov}\left\{G\left(e^{j \omega}, \hat{\theta}_{N}^{n}\right)\right\} \approx \frac{1}{N} \frac{\Phi_{\nu}(\omega)}{\Phi_{u}(\omega)} \sum_{k=0}^{n-1}\left|\mathcal{B}_{k}\left(e^{j \omega}\right)\right|^{2}
$$

and

$$
\operatorname{Cov}\left\{H\left(e^{j \omega}, \hat{\theta}_{N}^{n}\right)\right\} \approx \frac{\left|H\left(e^{j \omega}, \theta_{o}^{n}\right)\right|^{2}}{N} \sum_{k=0}^{n-1}\left|\mathcal{B}_{k}\left(e^{j \omega}\right)\right|^{2}
$$

which explicitly shows, via the $\sum_{k=0}^{n-1}\left|\mathcal{B}_{k}\left(e^{j \omega}\right)\right|^{2}=K_{n}(\omega, \omega)$ term, how the choice of fixed zeros $\left\{\xi_{k}\right\}$ in the noise model influences the sensitivity of the final transfer function estimation to measurement noise; again, see Fig. 4. Note that as per the previous section on fixed denominator model structures, these new expressions contain the results for equation error structures presented in [22] as a special case of $\xi_{k}=0$ since the latter implies $\sum_{k=0}^{n-1}\left|\mathcal{B}_{k}\left(e^{j \omega}\right)\right|^{2}=n$ so that (78) becomes (64). 
To conclude this section, it is interesting to note two important cases where fixed-zero noise models in ARX structures are implicitly involved, albeit via alternate motivations. Firstly, consider the case of employing a standard ARX structure save that, in the interests of concentrating attention on certain frequency regions [46], the common practice of data prefiltering is employed in such a way that [denoting the filtering action by the transfer function $F(q)$ ] the ARX model structure being employed becomes

$$
A\left(q, \theta^{n}\right) F(q) y_{t}=B\left(q, \theta^{n}\right) F(q) u_{t}+e_{t} .
$$

In this case, if the filter $F(q)$ is all pole of the form $F(q)=$ $1 / D_{n}(q)$ then this model structure is clearly identical to the model structure (63) with fixed-zero noise model and for which it was shown in Figs. 7-8 that the approximation (64) can be quite poor if the number of prefilter poles is an appreciable proportion of the model order. This leads to the conclusion that whenever all-pole prefilters are used with ARX structures, then the improved approximation (78) is preferable.

Finally, in the interests of numerical conditioning when sampling well beyond the Nyquist rate, the so-called " $\delta$ operator" model structure is an option [30], [7]. Such structures, when in equation error form as presented in [30], appear as

$$
\frac{A\left(\delta, \theta^{n}\right)}{D_{n}(\delta)} y_{t}=\frac{B\left(\delta, \theta^{n}\right)}{D_{n}(\delta)} u_{t}+e_{t}
$$

where with $\Delta$ equal to the sampling period in seconds, $\delta$ is defined as

$$
\delta \triangleq \frac{q-1}{\Delta}
$$

and

$$
\begin{aligned}
& A(\delta)=a_{0}+a_{1} \delta+a_{2} \delta^{2}+\cdots+a_{n-1} \delta^{n-1}+\delta^{n} \\
& B(q)=b_{0}+b_{1} \delta+b_{2} \delta^{2}+\cdots+b_{n-1} \delta^{n-1}
\end{aligned}
$$

with

$$
D_{n}(\delta)=\prod_{k=1}^{n}\left(\delta-\tilde{\xi}_{k}\right)
$$

and now the $\tilde{\xi}_{k}$ are chosen to all lie in the disc $\tilde{\mathbf{D}} \triangleq\{z \in$ $C:|z+1 / \Delta|<1 / \Delta\}$. Aside from numerical considerations, another dividend of using this model structure is that since the $\delta$ operator is the Euler differencing approximation to the differentiation operator, the ensuing estimates can be interpreted as an approximation of the underlying continuoustime system. Again, this depends on sampling well beyond the Nyquist rate [32].

In the $q$ operator case and in the absence of noise model knowledge, it is natural to simply choose $D_{n}(q)=q^{n}$. However, when operating in a $\delta$ operator framework it is not natural to make the specific equivalent choice $D_{n}(\delta)=$ $(\delta+1 / \Delta)^{n}$, since a key philosophy underlying the use of $\delta$ operators is that of more closely approaching continuous-time intuition, wherein the precise value of $\Delta$ is incidental. Other considerations therefore come into play in the choice of the zeros of $D_{n}(\delta)$, for which [30] provides a discussion.

In this setting, attention is therefore focused on a model structure (81) which is (modulo numerical issues) equivalent to the shift operator model (63) with fixed noise model zeros not at the origin, but at the locations $\xi_{k}=\Delta \tilde{\xi}_{k}+1$. The most accurate quantification of the variability of the ensuing $\delta$ operator-based estimates will therefore by given by the new approximation (78). These $\delta$ operator inspired asymptotic variability issues were first prescienced in [10].

\section{CONCLUSION}

This paper has provided an exposition of the previously unsuspected intrinsic nature of orthonormal bases in the study of certain least squares estimation problems. In works [25], [22], and [20] preceding this paper, the bases have been present in the special form corresponding to $\xi_{k}=0$ of $\mathcal{B}_{n}\left(e^{j \omega}\right)=e^{-j \omega n}$ so that they have been hidden since then $\left|\mathcal{B}_{n}\left(e^{j \omega}\right)\right|^{2}=\left|e^{-j \omega n}\right|^{2}=1$. However, as shown for the first time here in a completely general setting, they become apparent for certain structures with fixed pole or zero terms where $\left|D_{n}\left(e^{j \omega}\right)\right|^{2} \neq 1$, and hence whose changing nature with respect to increasing $n$ cannot be ignored in arguments that are asymptotic in $n$. This paper has shown that the analytic key to circumventing this difficulty is to develop and apply results that generalize certain Fourier convergence and asymptotic Toeplitz matrix properties to the case of an underlying general rational orthonormal basis, of which the classical trigonometric basis $\left\{e^{-j \omega n}\right\}$ is a special case.

As a final comment, it is important to acknowledge that the orthonormal basis ideas used in this paper have a very long history. The study of the formulation (25) in mathematical literature can be traced back at least as far as [28], [42], and the engineering applications (25) seem to originate in [17], although it clear that Wiener was aware of the practical relevance of (25) somewhat earlier [19]. In more recent times, the list of works employing specialized "Laguerre" or "twoparameter Kautz" forms of (25) is immense, but a partial list for readers interested in further investigations includes [29], [4], [38], [36], [26], [44], [45], [37], [15], [6], [36], [18]. However, a key feature discriminating this latter work from that in the current paper is that in pre-existing studies the orthonormal bases were explored as an implementational option while here, they have been employed purely as an analysis tool, and this tool is applicable to model structures that are not $a b$ initio formulated in terms of them.

\section{APPENDIX I \\ AUXILIARY RESULTS}

Lemma A.1: Let $\left\{\nu_{t}\right\}$ be a stationary stochastic process with associated covariance function $R_{\nu}(\tau)$ and spectral density $\Phi_{\nu}(\omega)$ satisfying

$$
\Phi_{\nu}(\omega)=\sum_{\tau=-\infty}^{\infty} R_{\nu}(\tau) e^{-j \omega \tau}, \quad \sum_{\tau=-\infty}^{\infty}\left|R_{\nu}(\tau)\right|<\infty
$$

and suppose $\left\{u_{t}\right\}$ is a quasi-stationary process with associated spectral density $\Phi_{u}(\omega)$. Then with $\psi_{t}=\Gamma_{n}(q) u_{t}$ and $\Gamma_{n}(q)$ 
defined by (32)

$$
\begin{aligned}
\lim _{N \rightarrow \infty} & \frac{1}{N} \sum_{k=0}^{N-1} \sum_{m=0}^{N-1} \mathbf{E}\left\{\psi_{k} \psi_{m}^{T}\right\} \mathbf{E}\left\{\nu_{k} \nu_{m}\right\} \\
= & \frac{1}{2 \pi} \int_{-\pi}^{\pi} \Gamma(\omega) \Gamma^{*}(\omega) \Phi_{u}(\omega) \Phi_{\nu}(\omega) d \omega
\end{aligned}
$$

Proof: Without loss of generality assume $u_{t}=0$ for $t<0$ so that

$$
\begin{aligned}
\lim _{N \rightarrow \infty} & \frac{1}{N} \sum_{k=0}^{N-1} \sum_{m=0}^{N-1} \mathbf{E}\left\{\psi_{k} \psi_{m}^{T}\right\} \mathbf{E}\left\{\nu_{k} \nu_{m}\right\} \\
= & \lim _{N \rightarrow \infty} \frac{1}{2 \pi N} \int_{-\pi}^{\pi} \Phi_{\nu}(\omega) \sum_{k=0}^{N-1} \sum_{m=0}^{N-1} \mathbf{E}\left\{\psi_{k} \psi_{m}^{T}\right\} \\
& \cdot e^{j \omega(k-m)} d \omega \\
= & \lim _{N \rightarrow \infty} \frac{1}{2 \pi} \int_{-\pi}^{\pi} \Phi_{\nu}(\omega) \sum_{\tau=1-N}^{N-1} \underbrace{\left(\frac{1}{N} \sum_{k=0}^{N-1} \mathbf{E}\left\{\psi_{k} \psi_{k-|\tau|}^{T}\right\}\right)}_{R_{N}(\tau)} \\
& \cdot e^{j \omega \tau} d \omega .
\end{aligned}
$$

Therefore, defining

$$
\begin{aligned}
R_{\psi}(\tau) & =\lim _{N \rightarrow \infty} \frac{1}{N} \sum_{k=0}^{N-1} \mathbf{E}\left\{\psi_{k} \psi_{k-|\tau|}^{T}\right\} \\
& =\frac{1}{2 \pi} \int_{-\pi}^{\pi} \Gamma_{n}(\omega) \Gamma_{n}^{*}(\omega) \Phi_{u}(\omega) e^{-j \omega \tau} d \omega
\end{aligned}
$$

leads to (the absolute value inequalities following are to be interpreted component-wise in the matrix quantities involved)

$$
\begin{aligned}
& \mid \frac{1}{2 \pi} \int_{-\pi}^{\pi} \Gamma(\omega) \Gamma^{*}(\omega) \Phi_{u}(\omega) \Phi_{\nu}(\omega) d \omega \\
& \quad-\frac{1}{N} \sum_{k=0}^{N-1} \sum_{m=0}^{N-1} \psi_{k} \psi_{m}^{T} R_{\nu}(k-m) \mid \\
&=\mid \frac{1}{2 \pi} \int_{-\pi}^{\pi} \Phi_{\nu}(\omega)\left[\left(\sum_{\tau=1-N}^{N-1} R_{\psi}(\tau)-R_{N}(\tau)\right)\right. \\
&\left.\quad+\sum_{|\tau| \geq N} R_{\psi}(\tau)\right] e^{j \omega \tau} d \omega \mid \\
& \leq \sum_{\tau=1-N}^{N-1}\left|R_{\psi}(\tau)-R_{N}(\tau)\right|\left|R_{\nu}(\tau)\right| \\
&+\left|\sum_{|\tau| \geq N} R_{\psi}(\tau) R_{\nu}(\tau)\right| \\
& \leq\left(\max _{|\tau|<N}\left|R_{\psi}(\tau)-R_{N}(\tau)\right| \sum_{\tau=1-N}^{N-1}\left|R_{\nu}(\tau)\right|\right. \\
&+\left(\sup _{|\tau| \geq N}\left|R_{\nu}(\tau)\right| \sum_{|\tau| \geq N}\left|R_{\psi}(\tau)\right| .\right.
\end{aligned}
$$

However, by the quasi-stationarity assumption on $\left\{u_{t}\right\}$, then $\lim _{N \rightarrow \infty} R_{N}(\tau)=R_{\psi}(\tau)$. So for sufficiently large $N$ then $\max _{|\tau|<N}\left|R_{\psi}(\tau)-R_{N}(\tau)\right|$ can be made arbitrarily small, and since also by assumption $\Sigma_{\tau}\left|R_{\nu}(\tau)\right|<\infty$, then the first term in the above overbound tends to zero with increasing $N$. For the second term, note that since by the assumption that $\Sigma_{\tau}\left|R_{\nu}(\tau)\right|<\infty$, then $\limsup _{\tau \rightarrow \infty}\left|R_{\nu}(\tau)\right|=0$ so that for large enough $N$, then $\sup _{|\tau| \geq N}\left|R_{\nu}(\tau)\right|$ can be made arbitrarily small. As well, by the quasi-stationarity assumption on $\left\{u_{t}\right\}$, which implies that $\Sigma_{\tau=-\infty}^{\infty} R_{\psi}(\tau)$ exists componentwise, then $\limsup \sup _{N \rightarrow \infty} \Sigma_{|\tau| \geq N}\left|R_{\psi}(\tau)\right|=0$, which completes the proof.

Lemma A.2: Suppose that $\left\{u_{t}\right\}$ and $\left\{e_{t}\right\}$ are both realizations of zero mean Gaussian distributed stationary stochastic processes and the orthonormal model structure (27) is employed. Then regardless of whether the data is collected in open or closed loop, and with no approximation caused by neglecting an undermodeling-induced term, the matrix $Q_{n}$ defined in (11) obeys

$$
Q_{n} \sim M_{n}\left(\Phi_{u}(\omega) \Phi_{\varepsilon}\left(\omega, \theta_{o}^{n}\right)\right)
$$

as $n \rightarrow \infty$ where the matrix formulation $M_{n}$ is defined in (31) and $\Phi_{\varepsilon}\left(\omega, \theta_{o}^{n}\right)$ is the spectral density of the prediction residuals $\left\{\varepsilon_{t}\left(\theta^{n}\right)\right\}$ defined in (6) and evaluated at $\theta^{n}=\theta_{o}^{n}$ defined in (31).

Proof: From the definition (11) after using the stationarity assumption and the change of variable $\tau=k+m$ and regrouping terms

$$
\begin{aligned}
Q_{n} & =\lim _{N \rightarrow \infty} \sum_{\tau=-N}^{N}\left(1-\frac{|\tau|}{N}\right) \mathbf{E}\left\{\psi_{k} \psi_{k-\tau}^{T} \varepsilon_{k}\left(\theta_{o}^{n}\right) \varepsilon_{k-\tau}\left(\theta_{o}^{n}\right)\right\} \\
& =\sum_{\tau=-\infty}^{\infty} \mathbf{E}\left\{\psi_{k} \psi_{k-\tau}^{T} \varepsilon_{k}\left(\theta_{o}^{n}\right) \varepsilon_{k-\tau}\left(\theta_{o}^{n}\right)\right\}
\end{aligned}
$$

where the properties of Cesàro means have been used in progressing to the last line. Using the Gaussianity assumption and the formula for fourth moments of jointly Gaussian random variables [43]

$$
\begin{aligned}
\mathbf{E}\left\{\psi_{k} \psi_{k-\tau}^{T} \varepsilon_{k}\left(\theta_{o}^{n}\right) \varepsilon_{k-\tau}\left(\theta_{o}^{n}\right)\right\} \\
=\mathbf{E}\left\{\psi_{k} \psi_{k-\tau}^{T}\right\} \mathbf{E}\left\{\varepsilon_{k}\left(\theta_{o}^{n}\right) \varepsilon_{k-\tau}\left(\theta_{o}^{n}\right)\right\} \\
\quad+\mathbf{E}\left\{\psi_{k} \varepsilon_{k-\tau}\left(\theta_{o}^{n}\right)\right\} \mathbf{E}\left\{\psi_{k-\tau}^{T} \varepsilon_{k}\left(\theta_{o}^{n}\right)\right\}
\end{aligned}
$$

where use is made of the fact that by the definition of $\theta_{o}^{n}$, $\mathbf{E}\left\{\psi_{k} \varepsilon_{k}\left(\theta_{o}^{n}\right)\right\}=0$. Furthermore (suppressing the dependence 
on $\theta_{o}^{n}$ and with $\delta(\cdot)$ denoting Kronecker delta)

$$
\begin{aligned}
& \sum_{\tau=-\infty}^{\infty} \mathbf{E}\left\{\psi_{k} \psi_{k-\tau}^{T}\right\} \mathbf{E}\left\{\varepsilon_{k} \varepsilon_{k-\tau}\right\} \\
&=\sum_{\ell=-\infty}^{\infty} \sum_{\tau=-\infty}^{\infty} \mathbf{E}\left\{\psi_{k} \psi_{k-\tau}^{T}\right\} \mathbf{E}\left\{\varepsilon_{k} \varepsilon_{k-\ell}\right\} \delta(\ell-\tau) \\
&=\sum_{\ell=-\infty}^{\infty} \sum_{\tau=-\infty}^{\infty} \mathbf{E}\left\{\psi_{k} \psi_{k-\tau}^{T}\right\} \mathbf{E}\left\{\varepsilon_{k} \varepsilon_{k-\ell}\right\} \\
& \cdot \frac{1}{2 \pi} \int_{-\pi}^{\pi} e^{j \omega(\ell-\tau)} d \omega \\
&=\frac{1}{2 \pi} \int_{-\pi}^{\pi} \sum_{\tau=-\infty}^{\infty} \mathbf{E}\left\{\psi_{k} \psi_{k-\tau}^{T}\right\} e^{-j \omega \tau} \\
& \cdot \sum_{\ell=-\infty}^{\infty} \mathbf{E}\left\{\varepsilon_{k} \varepsilon_{k-\ell}\right\} e^{j \omega \ell} d \omega \\
&= \frac{1}{2 \pi} \int_{-\pi}^{\pi} \Phi_{\psi}(\omega) \overline{\Phi_{\varepsilon}(\omega)} d \omega \\
&= \frac{1}{2 \pi} \int_{-\pi}^{\pi} \Gamma_{n}(\omega) \Gamma_{n}^{*}(\omega) \Phi_{u}(\omega) \Phi_{\varepsilon}(\omega) d \omega \\
&= M_{n}\left(\Phi_{u} \Phi_{\varepsilon}\right) .
\end{aligned}
$$

Using an identical line of argument

$$
\begin{aligned}
& \sum_{\tau=-\infty}^{\infty} \mathbf{E}\left\{\psi_{k} \varepsilon_{k-\tau}\right\} \mathbf{E}\left\{\psi_{k-\tau}^{T} \varepsilon_{k}\right\} \\
&=\frac{1}{2 \pi} \int_{-\pi}^{\pi} \sum_{\tau=-\infty}^{\infty} \mathbf{E}\left\{\psi_{k} \varepsilon_{k-\tau}\right\} e^{-j \omega \tau} \\
& \cdot \sum_{\ell=-\infty}^{\infty} \mathbf{E}\left\{\psi_{k-\ell}^{T} \varepsilon_{k}\right\} e^{j \omega \ell} d \omega \\
&= \frac{1}{2 \pi} \int_{-\pi}^{\pi} \sum_{\tau=-\infty}^{\infty} \mathbf{E}\left\{\psi_{k} \varepsilon_{k-\tau}\right\} e^{-j \omega \tau} \\
& \cdot \sum_{\ell=-\infty}^{\infty} \mathbf{E}\left\{\psi_{k}^{T} \varepsilon_{k-\ell}\right\} e^{-j \omega \ell} d \omega \\
&= \frac{1}{2 \pi} \int_{-\pi}^{\pi} \Phi_{\psi \varepsilon}(\omega) \Phi_{\psi \varepsilon}^{T}(\omega) d \omega \\
&= \frac{1}{2 \pi} \int_{-\pi}^{\pi} \Gamma_{n}(\omega) \Gamma_{n}^{T}(\omega) \Phi_{u \varepsilon}^{2}(\omega) d \omega \triangleq X_{n} .
\end{aligned}
$$

Now, in the case of all the poles $\left\{\xi_{k}\right\}$ being real, then since $\mathcal{B}_{k}\left(e^{j \omega}\right)=\overline{\mathcal{B}_{k}\left(e^{-j \omega}\right)}$

$$
\begin{aligned}
& \left|\frac{\Gamma_{n}^{*}(\omega) X_{n} \Gamma_{n}(\omega)}{K_{n}(\omega, \omega)}\right| \\
& \quad \leq \frac{\left\|\Phi_{u \varepsilon}\right\|_{\infty}}{2 \pi K_{n}(\omega, \omega)} \int_{-\pi}^{\pi}\left|K_{n}(\lambda, \omega) \| K_{n}(-\omega, \lambda)\right| d \lambda .
\end{aligned}
$$

However, in [35] it is shown that expressions of this form tend to zero with increasing $n$ provided all the poles $\left\{\xi_{k}\right\}$ are chosen within the open disc $\mathbf{D}$. This same result can also be shown to hold for the $\left\{\xi_{k}\right\}$ being complex, but at the expense of considerably more involved arithmetic which is not appropriate to document here. Therefore, since $Q_{n}=$
$M_{n}\left(\Phi_{u} \Phi_{\varepsilon}\right)+X_{n}$ and it has just been established that $X_{n} \sim 0$ as $n \rightarrow \infty$, then $Q_{n} \sim M_{n}\left(\Phi_{u} \Phi_{\varepsilon}\right)$ as $n \rightarrow \infty$.

Lemma A.3: Let $F(\omega)$ be a continuous $s \times s$ matrix-valued function defined on $[-\pi, \pi]$. Then

$$
\lim _{n \rightarrow \infty} \frac{1}{K_{n}(\omega, \omega)}\left[\Gamma_{n}^{*}(\omega) \otimes I_{s}\right] M_{n}(F)\left[\Gamma_{n}(\omega) \otimes I_{s}\right]=F(\omega)
$$

component-wise in $F(\omega)$ and where $M_{n}(F)$ is defined by (67) with $\Gamma_{n}(\omega)$ being the generalized form (32).

Proof: Using the algebraic properties of Kronecker tensor product [3] and the definition (67) of $M_{n}(F)$

$$
\begin{aligned}
& {\left[\Gamma_{n}^{*}(\omega) \otimes I_{s}\right] M_{n}(F)\left[\Gamma_{n}(\omega) \otimes I_{s}\right]} \\
& =\left[\Gamma_{n}^{*}(\omega) \otimes I_{s}\right]\left[\frac{1}{2 \pi} \int_{-\pi}^{\pi}\left[\Gamma_{n}(\lambda) \otimes I_{s}\right]\right. \\
& \left.\cdot F(\lambda)\left[\Gamma_{n}^{*}(\lambda) \otimes I_{s}\right] d \lambda\right] \times\left[\Gamma_{n}(\omega) \otimes I_{s}\right] \\
& =\frac{1}{2 \pi} \int_{-\pi}^{\pi}\left[\Gamma_{n}^{*}(\omega) \otimes I_{s}\right]\left[\Gamma_{n}(\lambda) \Gamma_{n}^{*}(\lambda) \otimes F(\lambda)\right] \\
& \quad \cdot\left[\Gamma_{n}(\omega) \otimes I_{s}\right] d \lambda \\
& =\frac{1}{2 \pi} \int_{-\pi}^{\pi}\left|\Gamma_{n}^{*}(\omega) \Gamma_{n}(\lambda)\right|^{2} \otimes F(\lambda) d \lambda .
\end{aligned}
$$

Use of the result (50) on noting that the above expression is a matrix made up of terms of the form $\Gamma_{n}^{*}(\omega) M_{n}\left(F_{m n}\right) \Gamma_{n}(\omega)$ with $F_{m n}(\lambda)$ being the $m, n$th scalar entry of $F(\lambda)$ and with $M_{n}(f)$ defined by (31), (32) then completes the proof.

Lemma A.4: With the definition of the generalized Toeplitz matrix being expanded to that of generalized block-Toeplitz matrix by (74), then for $F, G$ any $s \times s$ complex matrix-valued functions which have entries which are Lipschitz continuous of order $\varepsilon>0$

$$
\begin{aligned}
\lim _{n \rightarrow \infty} & K_{n}^{-1}(\omega, \omega)\left[\Gamma_{n}^{*}(\omega) \otimes I_{s}\right] \\
\cdot & {\left[M_{n}(F) M_{n}(G)-M_{n}(F G)\right]^{*} } \\
\times & {\left[M_{n}(F) M_{n}(G)-M_{n}(F G)\right] } \\
\cdot & {\left[\Gamma_{n}(\omega) \otimes I_{s}\right]=0 }
\end{aligned}
$$

are component-wise.

Proof: Using the formulation (74)

$$
\begin{aligned}
{\left[M_{n}(F)\right.} & \left.M_{n}(G)\left[\Gamma_{n}(\omega) \otimes I_{s}\right]\right]_{r s+k, t} \\
= & \frac{1}{4 \pi^{2}} \int_{-\pi}^{\pi} \int_{-\pi}^{\pi}\left[\Gamma_{n}(\lambda) K_{n}(\omega, \sigma) K_{n}(\sigma, \lambda)\right. \\
= & \frac{1}{4 \pi^{2}} \int_{-\pi}^{\pi} \int_{-\pi}^{\pi} \mathcal{B}_{r}(\lambda) K_{n}(\omega, \sigma) K_{n}(\sigma, \lambda) \\
& \cdot[F(\lambda) G(\sigma)]_{k, t} d \lambda d \sigma \\
= & \frac{1}{4 \pi^{2}} \sum_{\ell=0}^{s-1} \int_{-\pi}^{\pi} \int_{-\pi}^{\pi} \mathcal{B}_{r}(\lambda) K_{n}(\omega, \sigma) K_{n}(\sigma, \lambda) \\
& \times[F(\lambda)]_{k, \ell}[G(\sigma)]_{\ell, t} d \lambda d \sigma \\
& =\sum_{\ell-1}\left[M_{n}\left(F_{k, \ell}\right) M_{n}\left(G_{\ell, t}\right) \Gamma_{n}(\omega)\right]_{r} .
\end{aligned}
$$


Also, by a similar argument

$$
\left[M_{n}(F G)\left[\Gamma_{n}(\omega) \otimes I_{s}\right]\right]_{r s+k, t}=\sum_{\ell=0}^{s-1}\left[M_{n}\left(F_{k, \ell} G_{\ell, t}\right) \Gamma_{n}(\omega)\right]_{r}
$$

Therefore, defining $\Delta_{n} \triangleq M_{n}(F) M_{n}(G)-M_{n}(F G)$ leads to

$$
\begin{aligned}
& {\left[\left[\Gamma_{n}^{*}(\omega) \otimes I_{s}\right] \Delta_{n}^{*} \Delta_{n}\left[\Gamma_{n}(\omega) \otimes I_{s}\right]\right]_{p, t} } \\
&=\sum_{r=0}^{n-1} \sum_{k=0}^{s-1} \overline{\left[\Delta_{n}\left[\Phi_{n}(\omega) \otimes I_{s}\right]\right]_{r s+k, p}} \\
& \cdot\left[\Delta_{n}\left[\Phi_{n}(\omega) \otimes I_{s}\right]\right]_{r s+k, t} \\
&= \sum_{r=0}^{n-1} \sum_{k, \ell, v=0}^{s-1} \\
& \cdot \overline{\left[\left[M_{n}\left(F_{k, \ell}\right) M_{n}\left(G_{\ell, p}\right)-M_{n}\left(F_{k, \ell}, G_{\ell, p}\right)\right] \Gamma_{n}(\omega)\right]_{r}} \\
& \times\left[\left[M_{n}\left(F_{k, \ell}\right) M_{n}\left(G_{\ell, t}\right)-M_{n}\left(F_{k, \ell}, G_{\ell, t}\right)\right] \Gamma_{n}(\omega)\right]_{r} .
\end{aligned}
$$

Now, when $p=t$, then the scalar result (44) gives that this expression divided by $K_{n}(\omega, \omega)$ tends to zero as $n \rightarrow$ $\infty$. When $p \neq t$ it is first necessary to upper-bound the above expression using the Cauchy-Schwarz inequality as $\left|\Gamma_{n}^{*} A B \Gamma_{n}\right|^{2} \leq\left|\Gamma_{n}^{*} A A^{*} \Gamma_{n}\right|\left|\Gamma_{n}^{*} B^{*} B \Gamma_{n}\right|$ before again using the scalar result (44) to conclude that this overbound, and hence the expression of interest also tends to zero when divided by $K_{n}(\omega, \omega)$ as $n \rightarrow \infty$.

Lemma A.5: With the property (A2) being understood as the defining feature of the relationship $M_{n}(F) \sim M_{n}(G)$ as $n \rightarrow \infty$ for generalized block-Toeplitz matrices defined by (74), then for $F(\omega)$ any Lipschitz continuous matrix-valued function that is invertible

$$
M_{n}^{-1}(F) \sim M_{n}\left(F^{-1}\right) \quad \text { as } n \rightarrow \infty
$$

Proof:

$$
\begin{aligned}
{\left[\Gamma_{n}^{*}(\omega) \otimes\right.} & \left.I_{s}\right]\left[M_{n}^{-1}(F)-M_{n}\left(F^{-1}\right)\right]^{T} \\
& \times\left[M_{n}^{-1}(F)-M_{n}\left(F^{-1}\right)\right]\left[\Gamma_{n}(\omega) \otimes I_{s}\right] \\
= & {\left[\Gamma_{n}^{*}(\omega) \otimes I_{s}\right]\left[I-M_{n}(F) M_{n}\left(F^{-1}\right)\right]^{T} M_{n}^{-T}(F) } \\
& \cdot M_{n}^{-1}(F) \times\left[I-M_{n}(F) M_{n}\left(F^{-1}\right)\right]\left[\Gamma_{n}(\omega) \otimes I_{s}\right]
\end{aligned}
$$

However, by Lemma A.4 $M_{n}(F) M_{n}\left(F^{-1}\right) \sim M_{n}(I)=I$ as $n \rightarrow \infty$ so that use of the Cauchy-Schwarz inequality and the bound $\left\|M_{n}\left(F^{-1}\right)\right\| \leq\left\|F^{-1}\right\|_{\infty}$ completes the proof.

\section{REFERENCES}

[1] IEEE Trans. Automat. Contr. (Special Issue on System Identification for Robust Control Design), 1992.

[2] R. Bitmead, M. Gevers, and V. Wertz, Adaptive Optimal Control, The Thinking Man's GPC. Englewood Cliffs, NJ: Prentice-Hall, 1990.

[3] J. W. Brewer, "Kronecker products and matrix calculus in system theory," IEEE Trans. Circuits and Syst., vol. 25, pp. 772-781, 1978.

[4] P. W. Broome, "Discrete orthonormal sequences," J. Assoc. Computing Machinery, vol. 12, pp. 151-168, 1965.

[5] P. Caines, Linear Stochastic Systems. New York: Wiley, 1988.

[6] P. R. Clement, "Laguerre functions in signal analysis and parameter identification," J. Franklin Inst., vol. 313, pp. 85-95, 1982.

[7] A. Feuer and R. Middleton, "Conditioning of LMS algorithms with fast sampling," IEEE Trans. Signal Processing, vol. 43, pp. 1978-1981, 1995.
[8] M. Gevers, L. Ljung, and P. M. J. Van den Hof, "Asymptotic variance expressions for closed-loop identification and their relevance in identification for control," in Proc. 11th IFAC Symp. Syst. Identification, 1997, pp. 1449-1454.

[9] G. Golub and C. V. Loan, Matrix Computations. Baltimore, MD: Johns Hopkins Univ. Press, 1989.

[10] G. Goodwin, M. Gevers, and D. Mayne, "Bias and variance distribution in transfer function estimates," in Proc. 9th IFAC Symp. Identification and System Parameter Estimation, Budapest, 1991, pp. 952-957.

[11] U. Grenander and G. Szegö, Toeplitz Forms and Their Applications. Berkeley, CA: , Univ. California Press, 1958.

[12] L. Guo and L. Ljung, "The role of model validation for assessing the size of unmodeled dynamics," in Proc. 33rd Conf. Decision and Control, Dec. 1994, pp. 3894-3899.

[13] E. Hannan and D. Nicholls, "The estimation of the prediction error variance," J. Amer. Stat. Assoc., vol. 72, pp. 834-840, 1977.

[14] E. Hannan and B. Wahlberg, "Convergence rates for inverse Toeplitz matrix forms," J. Multivariate Anal., vol. 31, pp. 127-135, 1989.

[15] P. Heuberger, P. M. J. Van den Hof, and O. Bosgra, "A generalized orthonormal basis for linear dynamical systems," IEEE Trans. Automat. Contr., vol. 40, pp. 451-465, 1995.

[16] H. Hjalmarsson, M. Gevers, and F. De Bruyne, "For some model based control design criteria, closed loop identification gives better performance," Automatica, vol. 32, pp. 1659-1673, 1996.

[17] W. H. Kautz, "Network synthesis for specified transient response," Massachusetts Inst. Technol., Res. Lab. Electronics, Tech. Rep. 209, 1952.

[18] _ "Transient synthesis in the time domain," IRE Trans. Circuit Theory, vol. 1, pp. 29-39, 1954.

[19] Y. W. Lee, Statistical Theory of Communication. New York: Wiley, 1960.

[20] L. Ljung, System Identification: Theory for the User. Englewood Cliffs, NJ: Prentice-Hall, 1987.

[21] , "Identification in closed loop: Some aspects on direct and indirect approaches," in Proc. 11th IFAC Symp. System Identification, 1997, pp. 141-146.

[22] _Asymptotic variance expressions for identified black-box transfer function models," IEEE Trans. Automat. Contr., vol. AC-30, pp. 834-844, 1985

[23] L. Ljung and P. E. Caines, "Asymptotic normality of prediction error estimators for approximate system models," Stochastics, vol. 3, pp. 29-46, 1979.

[24] L. Ljung and B. Wahlberg, "Asymptotic properties of the least squares method for estimating transfer functions and disturbance spectra," Advances in Appl. Probability, vol. 24, pp. 412-440, 1992.

[25] L. Ljung and Z. D. Yuan, "Asymptotic properties of black-box identification of transfer functions," IEEE Trans. Automat. Contr., vol. 30, pp. 514-530, 1985.

[26] P. Mäkilä, "Laguerre methods and $H_{\infty}$ identification of continuous-time systems," Int. J. Contr., vol. 58, pp. 665-683, 1993.

[27] P. Mäkilä, J. Partington, and T. Gustafsson, "Worst-case control-relevant identification," Automatica, vol. 31, pp. 1799-1820, 1995.

[28] F. Malmquist, "Sur la détermination d'une classe de fonctions analytiques par leurs valeurs dans un ensemble donnè de points," Comptes Rendus du Sixiéme Congrès des mathématiciens scandinaves, Copenhagen, Denmark, 1925, pp. 253-259.

[29] J. Mendel, "A unified approach to the synthesis of orthonormal exponential functions useful in systems analysis," IEEE Trans. Syst. Science and Cybern., vol. 2, pp. 54-62, 1966.

[30] R. Middleton and G. Goodwin, Digital Estimation and Control: A Unified Approach. Englewood Cliffs, NJ: Prentice-Hall, 1990.

[31] B. Ninness, "Integral constraints on the accuracy of least squares estimation," Automatica, vol. 32, pp. 391-397, 1996.

[32] B. Ninness and G. Goodwin, Identification of Continuous Time Systems. New York: Kluwer, 1991. [33] $\frac{}{1995}$, "Estimation of model quality," Automatica, vol. 31, pp. 32-74,

[34] B. Ninness and F. Gustafsson, "A unifying construction of orthonormal bases for system identification," IEEE Trans. Automat. Contr., vol. 42, pp. 515-521, 1997.

[35] B. Ninness, H. Hjalmarsson, and F. Gustafsson, "Generalized Fourier and Toeplitz results for rational orthonormal bases," SIAM J. Contr. Optim., vol. 37, no. 2, pp. 429-460, 1999.

[36] Ü. Nurges, "Laguerre models in problems of approximation and identification," in Adaptive Systems. New York: Plenum Publishing, 1987, pp. 346-352.

[37] P. M. J. Van den Hof, P. S. C. Heuberger, and J. Bokor, "System identification with generalized orthonormal basis functions," Automatica, vol. 31, pp. 1821-1834, 1995. 
[38] D. Ross, "Orthonormal exponentials," IEEE Trans. Commun. and Electron., vol. 71, pp. 173-176, 1964

[39] M. Salgado, B. Ninness, and G. Goodwin, "Generalized expansion algorithm for identification of systems having colored noise," in Proc. Conf. Decision and Control, 1990.

[40] R. Smith and M. Dahleh, Eds., The Modeling of Uncertainty in Control Systems. New York: Springer Verlag, 1994.

[41] G. Szegö, Orthogonal Polynomials, vol. 23. New York: Colloquium, 1939.

[42] S. Takenaka, "On the orthogonal functions and a new formula of interpolation," Japanese J. Math., vol. II, pp. 129-145, 1925.

[43] T. Söderström and P. Stoica, System Identification. Englewood Cliffs, NJ: Prentice-Hall, 1989.

[44] B. Wahlberg, "System identification using Laguerre models," IEEE Trans. Automat. Contr., vol. 36, pp. 551-562, 1991.

[45] _ _ "System identification using Kautz models," IEEE Trans. Automat. Contr., vol. 39, pp. 1276-1282, 1994.

[46] B. Wahlberg and L. Ljung, "Design variables for bias distribution in transfer function estimation," IEEE Trans. Automat. Contr., vol. AC-31, pp. 134-144, 1986.

[47] H. Widom, Studies in Real and Complex Analysis, MAA Studies in Mathematics. Englewood Cliffs, NJ: Prentice-Hall, 1965.

[48] N. Wiener, The Fourier Integral and Certain of Its Applications. Cambridge, U.K.: Cambridge Univ. Press, 1933.

[49] Z. Yuan and L. Ljung, "Black box identification of multivariable transfer functions: Asymptotic properties and optimal input design," Int. J. Contr., vol. 40, pp. 233-256, 1984.

[50] Y. Zhu, "Black box identification of MIMO transfer functions: Asymptotic properties of prediction error models," Int. J. Adaptive Control and Signal Processing, vol. 3, pp. 357-373, 1989.

[51] A. Zygmund, Trigonometric Series. Cambridge, U.K.: Cambridge Univ. Press, 1959.

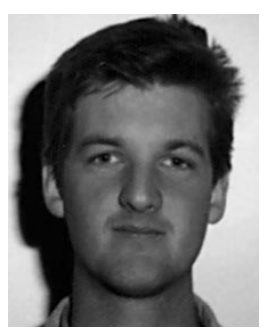

Brett Ninness (S'90-M'93) was born in 1963 in Singleton, Australia, and received the B.E, M.E., and Ph.D. degrees in electrical engineering from the University of Newcastle, Australia, in 1986, 1991, and 1994, respectively.

Since 1993, he has been a Lecturer and is currently a Senior Lecturer in the Department of Electrical and Computer Engineering at the University of Newcastle. He spent the latter half of 1997 enjoying a period as Guest Researcher in S3-Automatic Control, the Royal Institute of Technology, Stockholm, Sweden.

Dr. Ninness is an Associate Editor for Automatica, and is also a member of the Centre for Integrated Dynamics and Control (CIDAC), an Australian Government Special Research Centre.

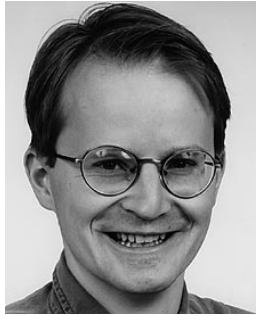

Håkan Hjalmarsson (M'98) was born in 1962. He received the M.S. degree in electrical engineering in 1988 and the Licentiate degree and the Ph.D. degree in automatic control in 1990 and 1993, respectively, all from Linköping University, Sweden.

During the academic year 1991-1992, he was a Visiting Lecturer in the Department of Chemical Engineering at the Californian Institute of Technology He was a Visiting Research Associate at CESAME, Louvain University, Belgium, during the academic year 1993-1994. In 1995, he visited the Department of Electrical Engineering and Computer Science, University of Newcastle, Australia, as a Research Fellow for six months. Currently, he is Associate Professor in the Department of Signals, Sensors and Systems, Royal Institute of Technology, Sweden. His research interests include system identification, signal processing and automated tuning of controllers.

Dr. Hjalmarsson is also an Associate Editor for Automatica.

Fredrik Gustafsson was born in 1964. He received the M.S. degree in electrical engineering in 1988 and the Ph.D degree in automatic control in 1992, both from Linköping University, Sweden.

$\mathrm{He}$ is an Associate Professor in Electrical Engineering at Linköping University. His research interests include statistical methods in system identification and signal processing, with applications to communication, avionic, and automotive systems. 